\title{
Hölder gradient estimates for a class of singular or degenerate parabolic equations
}

\author{
Cyril Imbert, Tianling Jin* and Luis Silvestre ${ }^{\dagger}$
}

September 6, 2016

\begin{abstract}
We prove interior Hölder estimate for the spatial gradients of the viscosity solutions to the singular or degenerate parabolic equation

$$
u_{t}=|\nabla u|^{\kappa} \operatorname{div}\left(|\nabla u|^{p-2} \nabla u\right),
$$

where $p \in(1, \infty)$ and $\kappa \in(1-p, \infty)$. This includes the from $L^{\infty}$ to $C^{1, \alpha}$ regularity for parabolic $p$-Laplacian equations in both divergence form with $\kappa=0$, and non-divergence form with $\kappa=2-p$. This work is a continuation of a paper by the last two authors [12].
\end{abstract}

\section{Introduction}

Let $1<p<\infty$ and $\kappa \in(1-p, \infty)$. We are interested in the regularity of solutions of

$$
u_{t}=|\nabla u|^{\kappa} \operatorname{div}\left(|\nabla u|^{p-2} \nabla u\right)
$$

When $\kappa=0$, this is the classical parabolic $p$-Laplacian equation in divergence form. This is the natural case in the context of gradient flows of Sobolev norms. Hölder estimates for the spatial gradient of their weak solutions (in the sense of distribution) were obtained by DiBenedetto and Friedman in [7] (see also Wiegner [24]).

When $\kappa=2-p$, the equation (1) is a parabolic homogeneous $p$-Laplacian equations. This is the most relevant case for applications to tug-of-war-like stochastic games with white noise, see Peres-Sheffield [20]. This equation has been studied by Garofalo [9], Banerjee-Garofalo [1, 2, 3], Does [8], Manfredi-Parviainen-Rossi [17, 18], Rossi [21], Juutinen [13], Kawohl-Krömer-Kurtz [14], Liu-Schikorra [16], Rudd [22], as well as the last two authors [12]. Hölder estimates for the spatial gradient of their solutions was proved in [12]. The solution of this equation is understood in the viscosity sense. The toolbox of methods that one can apply are completely different to the variational techniques used classically for $p$-Laplacian problems.

The equation (1) can be rewritten as

$$
u_{t}=|\nabla u|^{\gamma}\left(\Delta u+(p-2)|\nabla u|^{-2} u_{i} u_{j} u_{i j}\right),
$$

\footnotetext{
* Support in part by Hong Kong RGC grant ECS 26300716.

${ }^{\dagger}$ Support in part by NSF grants DMS-1254332 and DMS-1362525.
} 
where $\gamma=p+\kappa-2>-1$. In this paper, we prove Hölder estimates for the spatial gradients of viscosity solutions to (2) for $1<p<\infty$ and $\gamma \in(-1, \infty)$. Therefore, it provides a unified approach for all those $\gamma$ and $p$, including the two special cases $\gamma=0$ and $\gamma=p-2$ mentioned above.

The viscosity solutions to (2) with $\gamma>-1$ and $p>1$ falls into the general framework studied by Ohnuma-Sato in [19], which is an extension of the work of Barles-Georgelin [5] and Ishii-Souganidis [11] on the viscosity solutions of singular/degenerate parabolic equations. We postpone the definition of viscosity solutions of (2) to Section 5. For $r>0, Q_{r}$ denotes $B_{r} \times$ $\left(-r^{2}, 0\right]$, where $B_{r} \subset \mathbb{R}^{n}$ is the ball of radius $r$ centered at the origin.

Theorem 1.1. Let $u$ be a viscosity solution of (2) in $Q_{1}$, where $1<p<\infty$ and $\gamma \in(-1, \infty)$. Then there exist two constants $\alpha \in(0,1)$ and $C>0$, both of which depends only on $n, \gamma, p$ and $\|u\|_{L^{\infty}\left(Q_{1}\right)}$, such that

$$
\|\nabla u\|_{C^{\alpha}\left(Q_{1 / 2}\right)} \leq C .
$$

Also, the following Hölder regularity in time holds

$$
\sup _{(x, t),(x, s) \in Q_{1 / 2}} \frac{|u(x, t)-u(x, s)|}{|t-s|^{\frac{1+\alpha}{2-\alpha \gamma}}} \leq C .
$$

Note that $(1+\alpha) /(2-\alpha \gamma)>1 / 2$ for every $\alpha>0$ and $\gamma>-1$.

Our proof in this paper follows a similar structure as in [12], with some notable differences that we explain below. We use non-divergence techniques in the context of viscosity solutions. Theorem 1.1 tells us that these techniques are in some sense stronger than variational methods when dealing with the regularity of scalar $p$-Laplacian type equations. The weakness of these methods (at least as of now) is that they are ineffective for systems.

The greatest difficulty extending the result in [12] to Theorem 1.1 comes from the lack of uniform ellipticity. When $\gamma=0$, the equation (2) is a parabolic equation in non-divergence form with uniformly elliptic coefficients (depending on the solution $u$ ). Because of this, in [12], we use the theory developed by Krylov and Safonov, and other classical results, to get some basic uniform a priori estimates. This fact is no longer true for other values of $\gamma$. The first step in our proof is to obtain a Lipschitz modulus of continuity. That step uses the uniform ellipticity very strongly in [12]. In this paper we take a different approach using the method of Ishii and Lions [10]. Another step where the uniform ellipticity plays a strong role is in a lemma which transfers an oscillation bound in space, for every fixed time, to a space-time oscillation. In this paper that is achieved through Lemmas 4.4 and 4.5, which are considerably more difficult than their counterpart in [12]. Other, more minor, difficulties include the fact that the non-homogeneous right hand side forces us to work with a different scaling (See the definition of $Q_{r}^{\rho}$ by the beginning of Section 4).

In order to avoid some of the technical difficulties caused by the non-differentiability of viscosity solutions, we first consider the regularized problem (3) in the below, and then obtain uniform estimates so that we can pass to the limit in the end. For $\varepsilon \in(0,1)$, let $u$ be smooth and satisfy that

$$
\partial_{t} u=\left(|\nabla u|^{2}+\varepsilon^{2}\right)^{\gamma / 2}\left(\delta_{i j}+(p-2) \frac{u_{i} u_{j}}{|\nabla u|^{2}+\varepsilon^{2}}\right) u_{i j} .
$$

We are going to establish Lipschitz estimate and Hölder gradient estimates for $u$, which will be independent of $\varepsilon \in(0,1)$, in Sections 2, 3, 4. Then in Section 5, we recall the definition 
of viscosity solutions to (2), as well as their several useful properties, and prove Theorem 1.1 via approximation arguments. This idea of approximating the problem with a smoother one and proving uniform estimates is very standard.

Acknowledgement: Part of this work was done when T. Jin was visiting California Institute of Technology as an Orr foundation Caltech-HKUST Visiting Scholar. He would like to thank Professor Thomas Y. Hou for the kind hosting and discussions.

\section{Lipschitz estimates in the spatial variables}

The proof of Lipschitz estimate in [12] for $\gamma=0$ is based on a calculation that $|\nabla u|^{p}$ is a subsolution of a uniformly parabolic equation. We are not able to find a similar quantity for other nonzero $\gamma$. The proof we give here is completely different. It makes use of the Ishii-Lions' method [10]. However, we need to apply this method twice: first we obtain log-Lipschitz estimates, and then use this log-Lipschitz estimate and Ishii-Lions' method again to prove Lipschitz estimate. Moreover, the Lipschitz estimate holds for $\gamma>-2$ instead of $\gamma>-1$.

Lemma 2.1 (Log-Lipschitz estimate). Let $u$ be a smooth solution of (3) in $Q_{4}$ with $\gamma>-2$ and $\varepsilon \in(0,1)$. Then there exist two positive constants $L_{1}$ and $L_{2}$ depending only on $n, p, \gamma$ and $\|u\|_{L^{\infty}\left(Q_{4}\right)}$ such that for every $\left(t_{0}, x_{0}\right) \in Q_{1}$, we have

$$
u(t, x)-u(t, y) \leq L_{1}|x-y||\log | x-y||+\frac{L_{2}}{2}\left|x-x_{0}\right|^{2}+\frac{L_{2}}{2}\left|y-x_{0}\right|^{2}+\frac{L_{2}}{2}\left(t-t_{0}\right)^{2}
$$

for all $t \in\left[t_{0}-1, t_{0}\right]$ and $x, y \in B_{1}\left(x_{0}\right)$.

Proof. Without loss of generality, we assume $x_{0}=0$ and $t_{0}=0$. It is sufficient to prove that

$$
M:=\max _{-1 \leq t \leq 0, x, y \in \overline{B_{1}}}\left\{u(t, x)-u(t, y)-L_{1} \phi(|x-y|)-\frac{L_{2}}{2}|x|^{2}-\frac{L_{2}}{2}|y|^{2}-\frac{L_{2}}{2} t^{2}\right\}
$$

is non-positive, where

$$
\phi(r)= \begin{cases}-r \log r & \text { for } r \in\left[0, e^{-1}\right] \\ e^{-1} & \text { for } r \geq e^{-1} .\end{cases}
$$

We assume this is not true and we will exhibit a contradiction. In the rest of the proof, $t \in$ $[-1,0]$ and $x, y \in \bar{B}_{1}$ denote the points realizing the maximum defining $M$.

Since $M \geq 0$, we have

$$
L_{1} \phi(|x-y|)+\frac{L_{2}}{2}\left(|x|^{2}+|y|^{2}+t^{2}\right) \leq 2\|u\|_{L^{\infty}\left(Q_{4}\right)} .
$$

In particular,

$$
\phi(\delta) \leq \frac{2\|u\|_{L^{\infty}\left(Q_{4}\right)}}{L_{1}}, \quad \text { where } \delta=|a| \quad \text { and } \quad a=x-y
$$

and

$$
|t|+|x|+|y| \leq 6 \sqrt{\frac{\|u\|_{L^{\infty}\left(Q_{4}\right)}}{L_{2}}} .
$$


Hence, for $L_{2}$ large enough, depending only on $\|u\|_{L^{\infty}\left(Q_{4}\right)}$, we can ensure that $t \in(-1,0]$ and $x, y \in B_{1}$. We choose $L^{2}$ here and fix it for the rest of the proof. Thus, from now on $L_{2}$ is a constant depending only on $\|u\|_{L^{\infty}}$.

Choosing $L_{1}$ large, we can ensure that $\delta\left(<e^{-2}\right)$ is small enough to satisfy

$$
\phi(\delta) \geq 2 \delta \text {. }
$$

In this case, (4) implies

$$
\delta \leq \frac{\|u\|_{L^{\infty}\left(Q_{4}\right)}}{L_{1}}
$$

Since $t \in[-1,0]$ and $x, y \in B_{1}$ realizing the supremum defining $M$, we have that

$$
\begin{aligned}
& \nabla u(t, x)=L_{1} \phi^{\prime}(\delta) \hat{a}+L_{2} x \\
& \nabla u(t, y)=L_{1} \phi^{\prime}(\delta) \hat{a}-L_{2} y \\
& u_{t}(t, x)-u_{t}(t, y)=L_{2} t \\
& 0 \\
& {\left[\begin{array}{cc}
\nabla^{2} u(t, x)-\nabla^{2} u(t, y)
\end{array}\right] } \leq L_{1}\left[\begin{array}{cc}
Z & -Z \\
-Z & Z
\end{array}\right]+L_{2} I,
\end{aligned}
$$

where

$$
Z=\phi^{\prime \prime}(\delta) \hat{a} \otimes \hat{a}+\frac{\phi^{\prime}(\delta)}{\delta}(I-\hat{a} \otimes \hat{a}) \quad \text { and } \quad \hat{a}=\frac{a}{|a|}=\frac{x-y}{|x-y|} .
$$

For $z \in \mathbb{R}^{n}$, we let

$$
A(z)=I+(p-2) \frac{z_{i} z_{j}}{|z|^{2}+\varepsilon^{2}}
$$

and $q=L_{1} \phi^{\prime}(\delta) \hat{a}, X=\nabla^{2} u(t, x)$ and $Y=\nabla^{2} u(t, y)$. By evaluating the equation at $(t, x)$ and $(t, y)$, we have

$$
L_{2} t \leq\left(\left|q+L_{2} x\right|^{2}+\varepsilon^{2}\right)^{\frac{\gamma}{2}} \operatorname{Tr}\left(A\left(q+L_{2} x\right) X\right)-\left(\left|q-L_{2} y\right|^{2}+\varepsilon^{2}\right)^{\frac{\gamma}{2}} \operatorname{Tr}\left(A\left(q-L_{2} y\right) Y\right) .
$$

Whenever we write $C$ in this proof, we denote a positive constant, large enough depending only on $n, p, \gamma$ and $\|u\|_{L^{\infty}\left(Q_{4}\right)}$, which may vary from lines to lines. Recall that we have already chosen $L_{2}$ above depending on $\|u\|_{L^{\infty}}$ only.

Note that $|q|=L_{1}\left|\phi^{\prime}(\delta)\right|$. Choosing $L_{1}$ large enough, $\delta$ will be small, $\left|\phi^{\prime}(\delta)\right|$ will thus be large, and $|q| \gg L_{2}$. In particular,

$$
|q| / 2 \leq\left|q+L_{2} x\right| \leq 2|q| \quad \text { and } \quad|q| / 2 \leq\left|q-L_{2} y\right| \leq 2|q| .
$$

From (7) and the fact that $\phi^{\prime \prime}(\delta)<0$, we have

$$
\begin{array}{r}
X=\nabla^{2} u(t, x) \leq L_{1} \frac{\phi^{\prime}(\delta)}{\delta}(I-\hat{a} \otimes \hat{a})+L_{2} I \\
-Y=-\nabla^{2} u(t, y) \leq L_{1} \frac{\phi^{\prime}(\delta)}{\delta}(I-\hat{a} \otimes \hat{a})+L_{2} I
\end{array}
$$


Making use of (8), (9) and (10), we have

$$
\begin{aligned}
\operatorname{Tr}\left(A\left(q+L_{2} x\right) X\right) & =\left(\left|q+L_{2} x\right|^{2}+\varepsilon^{2}\right)^{-\frac{\gamma}{2}} L_{2} t+\left(\frac{\left|q-L_{2} y\right|^{2}+\varepsilon^{2}}{\left|q+L_{2} x\right|^{2}+\varepsilon^{2}}\right)^{\frac{\gamma}{2}} \operatorname{Tr}\left(A\left(q-L_{2} y\right) Y\right) \\
& \geq-C\left(|q|^{-\gamma}+L_{1} \frac{\phi^{\prime}(\delta)}{\delta}+1\right) .
\end{aligned}
$$

Therefore, it follows from (10) and the ellipticity of $A$ that

$$
|X| \leq C\left(|q|^{-\gamma}+L_{1} \frac{\phi^{\prime}(\delta)}{\delta}+1\right)
$$

Similarly,

$$
|Y| \leq C\left(|q|^{-\gamma}+L_{1} \frac{\phi^{\prime}(\delta)}{\delta}+1\right)
$$

Let

$$
B(z)=\left(|z|^{2}+\varepsilon\right)^{\gamma} A(z) .
$$

We get from (8) and (5) the following inequality

$$
-C \leq \operatorname{Tr}\left[B\left(q+L_{2} x\right) X\right]-\operatorname{Tr}\left[B\left(q-L_{2} y\right) Y\right] \leq T_{1}+T_{2}
$$

where

$$
\left.T_{1}=\operatorname{Tr}\left[B\left(q-L_{2} y\right)(X-Y)\right] \quad \text { and } \quad T_{2}=|X| \mid B\left(q+L_{2} x\right)-B\left(q-L_{2} y\right)\right) \mid .
$$

We first estimate $T_{2}$. Using successively (5), (9), (11) and mean value theorem, we get

$$
\begin{aligned}
T_{2} & \leq C|X||q|^{\gamma-1}|x+y| \\
& \leq C|X||q|^{\gamma-1} \\
& \leq C\left(|q|^{-\gamma}+\frac{L_{1} \phi^{\prime}(\delta)}{\delta}+1\right)|q|^{\gamma-1}, \\
& \leq C\left(|q|^{-1}+\frac{|q|^{\gamma}}{\delta}+|q|^{\gamma-1}\right) .
\end{aligned}
$$

We now turn to $T_{1}$. On one hand, evaluating (7) with respect to a vector of the form $(\xi, \xi)$, we get that for all $\xi \in \mathbb{R}^{d}$ we have

$$
(X-Y) \xi \cdot \xi \leq 2 L_{2}|\xi|^{2} .
$$

On the other hand, when we evaluate (7) with respect to $(\hat{a}, \hat{a})$, we get,

$$
(X-Y) \hat{a} \cdot \hat{a} \leq 4 L_{1} \phi^{\prime \prime}(\delta)+2 L_{2}
$$

The inequality (14) tells us that all eigenvalues of $(X-Y)$ are bounded above by a constant $C$. The inequality (15) tells us that there is at least one eigenvalue that is less than the negative number $4 L_{1} \phi^{\prime \prime}(\delta)+2 L_{2}$. Because of the uniform ellipticity of $A$, we obtain

$$
T_{1} \leq C|q|^{\gamma}\left(L_{1} \phi^{\prime \prime}(\delta)+1\right) \text {. }
$$


In view of the estimates for $T_{1}$ and $T_{2}$, we finally get from (12) that

$$
-L_{1} \phi^{\prime \prime}(\delta)|q|^{\gamma} \leq C\left(|q|^{\gamma}+|q|^{-1}+\frac{|q|^{\gamma}}{\delta}+|q|^{\gamma-1}+1\right)
$$

or equivalently,

$$
-L_{1} \phi^{\prime \prime}(\delta) \leq C\left(1+|q|^{-1-\gamma}+\frac{1}{\delta}+|q|^{-1}+|q|^{-\gamma}\right)
$$

Our purpose is to choose $L_{1}$ large in order to get a contradiction in (16).

Recall that we have the estimate $\delta \leq C / L_{1}$. From our choice of $\phi, \phi^{\prime}(\delta)>1$ for $\delta$ small and $-\phi^{\prime \prime}(\delta)=1 / \delta \geq c L_{1}$.

For $L_{1}$ sufficiently large, since $\gamma>-2$

$$
\begin{aligned}
C\left(1+|q|^{-1-\gamma}+|q|^{-1}+|q|^{-\gamma}\right) & \leq C\left(1+L_{1}^{-1-\gamma}+L_{1}^{-1}+L_{1}^{-\gamma}\right) \leq \frac{c}{2} L_{1}^{2}, \\
& \leq-\frac{1}{2} L_{1} \phi^{\prime \prime}(\delta) .
\end{aligned}
$$

The remaining term is handled because of the special form of the function $\phi$. We have

$$
-L_{1} \phi^{\prime \prime}(\delta)=\frac{L_{1}}{\delta}>\frac{2 C}{\delta}
$$

for $L_{1}$ sufficiently large.

Therefore, we reached a contradiction. The proof of this lemma is thereby completed.

By letting $t=t_{0}$ and $y=x_{0}$ in Lemma 2.1, and since $\left(x_{0}, t_{0}\right)$ is arbitrary, we have

Corollary 2.2. Let $u$ be a smooth solution of (3) in $Q_{4}$ with $\gamma>-2$ and $\varepsilon \in(0,1)$. Then there exists a positive constant $C$ depending only on $n, \gamma, p$ and $\|u\|_{L^{\infty}\left(Q_{4}\right)}$ such that for every $(t, x),(t, y) \in Q_{3}$ and $|x-y|<1 / 2$, we have

$$
|u(t, x)-u(t, y)| \leq C|x-y||\log | x-y|| .
$$

We shall make use of the above log-Lipschitz estimate and the Ishii-Lions' method [10] again to prove the following Lipschitz estimate.

Lemma 2.3 (Lipschitz estimate). Let $u$ be a smooth solution of (3) in $Q_{4}$ with $\gamma>-2$ and $\varepsilon \in(0,1)$. Then there exist two positive constants $L_{1}$ and $L_{2}$ depending only on $n, p, \gamma$ and $\|u\|_{L^{\infty}\left(Q_{4}\right)}$ such that for every $\left(t_{0}, x_{0}\right) \in Q_{1}$, we have

$$
u(t, x)-u(t, y) \leq L_{1}|x-y|+\frac{L_{2}}{2}\left|x-x_{0}\right|^{2}+\frac{L_{2}}{2}\left|y-x_{0}\right|^{2}+\frac{L_{2}}{2}\left(t-t_{0}\right)^{2}
$$

for all $t \in\left[t_{0}-1, t_{0}\right]$ and $x, y \in B_{1 / 4}\left(x_{0}\right)$. 
Proof. The proof of this lemma follows the same computations as that of Lemma 2.1, but we make use of the conclusion of Corollary 2.2 in order to improve our estimate.

Without loss of generality, we assume $x_{0}=0$ and $t_{0}=0$. As before, we define

$$
M:=\max _{-1 \leq t \leq 0, x, y \in B_{1}}\left\{u(t, x)-u(t, y)-L_{1} \phi(|x-y|)-\frac{L_{2}}{2}|x|^{2}-\frac{L_{2}}{2}|y|^{2}-\frac{L_{2}}{2} t^{2}\right\}
$$

is non-positive, where

$$
\phi(r)= \begin{cases}r-\frac{1}{2-\gamma_{0}} r^{2-\gamma_{0}} & \text { for } r \in[0,1] \\ 1-\frac{1}{2-\gamma_{0}} & \text { for } r \geq 1\end{cases}
$$

for some $\gamma_{0} \in(1 / 2,1)$.

We assume this is not true in order to obtain a contradiction. In the remaining of the proof of the lemma, $t \in[-1,0]$ and $x, y \in \bar{B}_{1 / 4}$ denote the points realizing the maximum defining $M$.

For the same reasons as in the proof of Lemma 2.1, the inequalities (4) and (5) also apply in this case. Thus, we can use the same choice of $L_{2}$, depending on $\|u\|_{L^{\infty}}$ only, that ensures $t \in(-1,0]$ and $x, y \in B_{1}$.

From Corollary 2.2, we already know that $u(t, x)-u(t, y) \leq C|x-y||\log | x-y||$. Since $M \geq 0$,

$$
L_{1} \phi(|x-y|)+\frac{L_{2}}{2}\left(|x|^{2}+|y|^{2}+t^{2}\right) \leq C|x-y||\log | x-y|| .
$$

In particular, we obtain an improvement of (5),

$$
|t|+|x|+|y| \leq C \sqrt{\frac{\delta|\log \delta|}{L_{2}}} .
$$

This gives us an upper bound for $|x+y|$ that we can use to improve (13).

$$
\begin{aligned}
T_{2} & \leq C|X||q|^{\gamma-1}|x+y|, \\
& \leq C\left(|q|^{-1}+\frac{|q|^{\gamma}}{\delta}+|q|^{\gamma-1}\right) \sqrt{\delta|\log \delta|},
\end{aligned}
$$

The estimate for $T_{1}$ stays unchanged. Hence, (16) becomes

$$
-L_{1} \phi^{\prime \prime}(\delta) \leq C\left(1+\sqrt{\delta|\log \delta|}\left(|q|^{-1}+|q|^{-1-\gamma}+\frac{1}{\delta}+|q|^{-\gamma}\right)\right) .
$$

Recall that $|q|=L_{1} \phi^{\prime}(\delta) \geq L_{1} / 2$ and $\phi^{\prime \prime}(\delta)=\left(\gamma_{0}-1\right) \delta^{-\gamma_{0}}$. Then,

$$
L_{1} \delta^{-\gamma_{0}} \leq C\left(1+\sqrt{\delta|\log \delta|}\left(1+L_{1}^{-1}+L_{1}^{-1-\gamma}+\delta^{-1}+L_{1}^{-\gamma}\right)\right)
$$

The term +1 inside the innermost parenthesis is there just to ensure that the inequality holds both for $\gamma<0$ and $\gamma>0$. Recalling that $\delta<C / L_{1}$, we obtain an inequality in terms of $L_{1}$ only.

$$
L_{1}^{1+\gamma_{0}} \leq C\left(1+L_{1}^{-1 / 2} \sqrt{\log L_{1}}\left(1+L_{1}^{-1}+L_{1}^{-1-\gamma}+L_{1}+L_{1}^{-\gamma}\right)\right)
$$

Choosing $L_{1}$ large, we arrive to a contradiction given that $1+\gamma_{0}>\max (1 / 2,-1 / 2-\gamma)$ since $\gamma_{0}>1 / 2$ and $\gamma>-2$. 
Again, by letting $t=t_{0}$ and $y=x_{0}$ in Lemma 2.3, and since $\left(x_{0}, t_{0}\right)$ is arbitrary, we have

Corollary 2.4. Let $u$ be a smooth solution of (3) in $Q_{4}$ with $\gamma>-2$ and $\varepsilon \in(0,1)$. Then there exists a positive constant $C$ depending only on $n, \gamma, p$ and $\|u\|_{L^{\infty}\left(Q_{4}\right)}$ such that for every $(t, x),(t, y) \in Q_{3}$ and $|x-y|<1$, we have

$$
|u(t, x)-u(t, y)| \leq C|x-y|
$$

\section{Hölder estimates in the time variable}

Using the Lipschitz continuity in $x$ and a simple comparison argument, we show that the solution of (3) is Hölder continuous in $t$.

Lemma 3.1. Let $u$ be a smooth solution of (3) in $Q_{4}$ with $\gamma>-1$ and $\varepsilon \in(0,1)$. Then there holds

$$
\sup _{t \neq s,(t, x),(s, x) \in Q_{1}} \frac{|u(t, x)-u(s, x)|}{|t-s|^{1 / 2}} \leq C,
$$

where $C$ is a positive constant depending only on $n, p, \gamma$ and $\|u\|_{L^{\infty}\left(Q_{4}\right)}$.

Remark 3.2. Deriving estimates in the time variable for estimates in the space variable by maximum principle techniques is classical. As far as viscosity solutions are concerned, the reader is referred to [4, Lemma 9.1, p. 317] for instance.

Proof. Let $\beta=\max (2,(2+\gamma) /(1+\gamma))$. We claim that for all $t_{0} \in[-1,0), \eta>0$, there exists $L_{1}>0$ and $L_{2}>0$ such that

$$
u(t, x)-u\left(t_{0}, 0\right) \leq \eta+L_{1}\left(t-t_{0}\right)+L_{2}|x|^{\beta}=: \varphi(t, x) \quad \text { for all }(t, x) \in\left[t_{0}, 0\right] \times \bar{B}_{1} .
$$

We first choose $L_{2} \geq 2\|u\|_{L^{\infty}\left(Q_{3}\right)}$ such that (19) holds true for $x \in \partial B_{1}$. We will next choose $L_{2}$ such that (19) holds true for $t=t_{0}$. In this step we shall use Corollary 2.4 that $u$ is Lipschitz

continuous with respect to the spatial variables. From Corollary $2.4,\|\nabla u\|_{L^{\infty}\left(Q_{3}\right)}$ is bounded depending on $\|u\|_{L^{\infty}\left(Q_{4}\right)}$ only. It is enough to choose

$$
\|\nabla u\|_{L^{\infty}\left(Q_{3}\right)}|x| \leq \eta+L_{2}|x|^{\beta}
$$

which holds true if

$$
L_{2} \geq \frac{\|\nabla u\|_{L^{\infty}\left(Q_{3}\right)}^{\beta}}{\eta^{\beta-1}} .
$$

We finally choose $L_{1}$ such that the function $\varphi(t, x)$ is a supersolution of an equation that $u$ is a solution. The inequality (19) thus follows from the comparison principle. We use a slightly different equation depending on whether $\gamma \leq 0$ or $\gamma>0$.

Let us start with the case $\gamma \leq 0$. In this case we will prove that $\varphi$ is a supersolution of the nonlinear equation (3). That is

$$
\varphi_{t}-\left(\varepsilon^{2}+|\nabla \varphi|^{2}\right)^{\gamma / 2}\left(\delta_{i j}+(p-2) \frac{\varphi_{i} \varphi_{j}}{\varepsilon^{2}+|\nabla \varphi|^{2}}\right) \varphi_{i j}>0 .
$$


In order to ensure this inequality, we choose $L_{1}$ so that

$$
L_{1}>(p-1)|\nabla \varphi|^{\gamma}\left|D^{2} \varphi\right| \geq\left(\varepsilon^{2}+|\nabla \varphi|^{2}\right)^{\gamma / 2}\left(\delta_{i j}+(p-2) \frac{\varphi_{i} \varphi_{j}}{\varepsilon^{2}+|\nabla \varphi|^{2}}\right) \varphi_{i j} .
$$

We chose the exponent $\beta$ so that when $\gamma \leq 0,|\nabla \varphi|^{\gamma}\left|D^{2} \varphi\right|=C L_{1}^{1+\gamma}$ for some constant $C$ depending on $n$ and $\gamma$. Thus, we must choose $L_{1}=C L_{2}^{1+\gamma}$ in order to ensure (20).

Therefore, still for the case $\gamma \leq 0, \beta=(2+\gamma) /(1+\gamma)$, and for any choice of $\eta>0$, using the comparison principle,

$$
\begin{aligned}
u(t, 0)-u\left(t_{0}, 0\right) & \leq \eta+C\left(\eta^{(1-\beta)}\|\nabla u\|_{L^{\infty}\left(Q_{3}\right)}^{\beta}+2\|u\|_{L^{\infty}\left(Q_{3}\right)}+\varepsilon\right)^{\gamma+1}\left(t-t_{0}\right) \\
& \leq \eta+C \eta^{-1}\|\nabla u\|_{L^{\infty}\left(Q_{3}\right)}^{\gamma+2}\left|t-t_{0}\right|+C\left(\|u\|_{L^{\infty}\left(Q_{3}\right)}+\varepsilon\right)^{\gamma+1}\left|t-t_{0}\right|
\end{aligned}
$$

By choosing $\eta=\|\nabla u\|_{L^{\infty}\left(Q_{3}\right)}^{\gamma / 2+1}\left|t-t_{0}\right|^{1 / 2}$, it follows that for $t \in\left(t_{0}, 0\right]$,

$$
u(t, 0)-u\left(t_{0}, 0\right) \leq C\left(\|\nabla u\|_{L^{\infty}\left(Q_{3}\right)}\right)^{\frac{\gamma+2}{2}}\left|t-t_{0}\right|^{1 / 2}+C\left(\|u\|_{L^{\infty}\left(Q_{3}\right)}+\varepsilon\right)^{\gamma+1}\left|t-t_{0}\right| .
$$

The lemma is then concluded in the case $\gamma \leq 0$.

Let us now analyze the case $\gamma>0$. In this case, we prove that $\varphi$ is a supersolution to a linear parabolic equation whose coefficients depend on $u$. That is

$$
\varphi_{t}-\left(\varepsilon^{2}+|\nabla u|^{2}\right)^{\gamma / 2}\left(\delta_{i j}+(p-2) \frac{u_{i} u_{j}}{\varepsilon^{2}+|\nabla u|^{2}}\right) \varphi_{i j}>0 .
$$

Since $\gamma>0$ and $\nabla u$ is known to be bounded after Corollary 2.4, we can rewrite the equation assumption

$$
\varphi_{t}-a_{i j}(t, x) \varphi_{i j}>0
$$

where the coefficients $a_{i j}(t, x)$ are bounded by

$$
\left|a_{i j}(t, x)\right| \leq C\left(\varepsilon+\|\nabla u\|_{L^{\infty}\left(Q_{3}\right)}\right)^{\gamma} .
$$

Since $\gamma>0$, we pick $\beta=2$ and $D^{2} \varphi$ is a constant multiple of $L_{2}$. In particular, we ensure that (22) holds if

$$
L_{1}>C\left(\varepsilon+\|\nabla u\|_{L^{\infty}\left(Q_{3}\right)}\right)^{\gamma} L_{2} .
$$

Therefore, for the case $\gamma>0, \beta=2$, and for any choice of $\eta>0$, using the comparison principle,

$$
u(t, 0)-u\left(t_{0}, 0\right) \leq \eta+C\left(\varepsilon+\|\nabla u\|_{L^{\infty}\left(Q_{3}\right)}\right)^{\gamma}\left(\eta^{-1}\|\nabla u\|_{L^{\infty}\left(Q_{3}\right)}^{2}+\|u\|_{L^{\infty}\left(Q_{3}\right)}\right)\left(t-t_{0}\right) .
$$

Choosing $\eta=\left(\varepsilon+\|\nabla u\|_{L^{\infty}\left(Q_{3}\right)}\right)^{\gamma / 2+1}\left(t-t_{0}\right)^{1 / 2}$, we obtain,

$$
\begin{aligned}
u(t, 0)-u\left(t_{0}, 0\right) \leq C\left(\varepsilon+\|\nabla u\|_{L^{\infty}\left(Q_{3}\right)}\right)^{\gamma / 2+1}\left(t-t_{0}\right)^{1 / 2}+ \\
C\left(\varepsilon+\|\nabla u\|_{L^{\infty}\left(Q_{3}\right)}\right)^{\gamma}\|u\|_{L^{\infty}\left(Q_{3}\right)}\left(t-t_{0}\right) .
\end{aligned}
$$

This finishes the proof for $\gamma>0$ as well. 


\section{Hölder estimates for the spatial gradients}

In this section, we assume that $\gamma>-1$ so that Corollary 2.4 and Lemma 3.1 holds, that is, the solution of (3) in $Q_{2}$ has uniform interior Lipschitz estimates in $x$ and uniform interior Hölder estimates in $t$, both of which are independent of $\varepsilon \in(0,1)$. For $\rho, r>0$, we denote

$$
Q_{r}=B_{r} \times\left(-r^{2}, 0\right], \quad Q_{r}^{\rho}=B_{r} \times\left(-\rho^{-\gamma} r^{2}, 0\right] .
$$

The cylinders $Q_{r}^{\rho}$ are the natural ones that correspond to the two-parameter family of scaling of the equation. Indeed, if $u$ solves (3) in $Q_{r}^{\rho}$ and we let $v(x, t)=\frac{1}{r \rho} u\left(r x, r^{2} \rho^{-\gamma} t\right)$, then

$$
v_{t}(t, x)=\left(|\nabla v|^{2}+\varepsilon^{2} \rho^{-2}\right)^{\gamma / 2}\left(\Delta v+(p-2) \frac{v_{i} v_{j}}{|\nabla v|^{2}+\varepsilon^{2} \rho^{-2}} v_{i j}\right) \quad \text { in } Q_{1} .
$$

If we choose $\rho \geq\|\nabla u\|_{L^{\infty}\left(Q_{1}\right)}+1$, we may assume that the solution of (3) satisfies $|\nabla u| \leq 1$ in $Q_{1}$.

We are going to show that $\nabla u$ is Hölder continuous in space-time at the point $(0,0)$. The idea of the proof in this step is similar to that in [12]. First we show that if the projection of $\nabla u$ onto the direction $e \in \mathbb{S}^{n-1}$ is away from 1 in a positive portion of $Q_{1}$, then $\nabla u \cdot e$ has improved oscillation in a smaller cylinder.

Lemma 4.1. Let $u$ be a smooth solution of (3) with $\varepsilon \in(0,1)$ such that $|\nabla u| \leq 1$ in $Q_{1}$. For every $\frac{1}{2}<\ell<1, \mu>0$, there exists $\tau_{1} \in\left(0, \frac{1}{4}\right)$ depending only on $\mu, n$, and there exist $\tau, \delta>0$ depending only on $n, p, \gamma, \mu$ and $\ell$ such that for arbitrary $e \in \mathbb{S}^{n-1}$, if

$$
\left|\left\{(x, t) \in Q_{1}: \nabla u \cdot e \leq \ell\right\}\right|>\mu\left|Q_{1}\right|
$$

then

$$
\nabla u \cdot e<1-\delta \text { in } Q_{\tau}^{1-\delta}
$$

and $Q_{\tau}^{1-\delta} \subset Q_{\tau_{1}}$

Proof. Let

$$
a_{i j}(q)=\left(|q|^{2}+\varepsilon^{2}\right)^{\gamma / 2}\left(\delta_{i j}+(p-2) \frac{q_{i} q_{j}}{|q|^{2}+\varepsilon^{2}}\right), q \in \mathbb{R}^{n}
$$

and denote

$$
a_{i j, m}=\frac{\partial a_{i j}}{\partial q_{m}}
$$

Differentiating (3) in $x_{k}$, we have

$$
\left(u_{k}\right)_{t}=a_{i j}\left(u_{k}\right)_{i j}+a_{i j, m} u_{i j}\left(u_{k}\right)_{m} .
$$

Then

$$
(\nabla u \cdot e-\ell)_{t}=a_{i j}(\nabla u \cdot e-\ell)_{i j}+a_{i j, m} u_{i j}(\nabla u \cdot e-\ell)_{m}
$$

and for

$$
v=|\nabla u|^{2}
$$


we have

$$
v_{t}=a_{i j} v_{i j}+a_{i j, m} u_{i j} v_{m}-2 a_{i j} u_{k i} u_{k j} .
$$

For $\rho=\ell / 4$, let

$$
w=\left(\nabla u \cdot e-\ell+\rho|\nabla u|^{2}\right)^{+} .
$$

Then in the region $\Omega_{+}=\left\{(x, t) \in Q_{1}: w>0\right\}$, we have

$$
w_{t}=a_{i j} w_{i j}+a_{i j, m} u_{i j} w_{m}-2 \rho a_{i j} u_{k i} u_{k j} .
$$

Since $|\nabla u|>\ell / 2$ in $\Omega_{+}$, we have in $\Omega_{+}$:

$$
\left|a_{i j, m}\right| \leq \begin{cases}c(p, n, \gamma) \ell^{-1}, & \text { if } \gamma \geq 0 \\ c(p, n, \gamma) \ell^{\gamma-1}, & \text { if } \gamma<0\end{cases}
$$

where $c(p, n, \gamma)$ is a positive constant depending only on $p, n$ and $\gamma$. By Cauchy-Schwarz inequality, it follows that

$$
w_{t} \leq a_{i j} w_{i j}+c_{1}(\ell)|\nabla w|^{2} \quad \text { in } \Omega_{+},
$$

where

$$
c_{1}(\ell)= \begin{cases}c_{0} \ell^{-\gamma-3}, & \text { if } \gamma \geq 0 \\ c_{0} \ell^{2 \gamma-3}, & \text { if } \gamma<0\end{cases}
$$

for some constant $c_{0}>0$ depending only on $p, \gamma, n$. Therefore, it satisfies in the viscosity sense that

$$
w_{t} \leq \tilde{a}_{i j} w_{i j}+c_{1}(\ell)|\nabla w|^{2} \quad \text { in } Q_{1}
$$

where

$$
\tilde{a}_{i j}(x)=\left\{\begin{array}{l}
a_{i j}(\nabla u(x)), \quad x \in \Omega_{+} \\
\delta_{i j}, \quad \text { elsewhere. }
\end{array}\right.
$$

Notice that since $\ell \in\left(\frac{1}{2}, 1\right), \tilde{a}_{i j}$ is uniformly elliptic with ellipticity constants depending only on $p$ and $\gamma$. We can choose $c_{2}(\ell)>0$ depending only on $p, \gamma, n$ and $\ell$ such that if we let

$$
W=1-\ell+\rho
$$

and

$$
\bar{w}=\frac{1}{c_{2}}\left(1-e^{c_{2}(w-W)}\right)
$$

then we have

$$
\bar{w}_{t} \geq \tilde{a}_{i j} \bar{w}_{i j} \quad \text { in } Q_{1}
$$

in the viscosity sense. Since $W \geq \sup _{Q_{1}} w$, then $\bar{w} \geq 0$ in $Q_{1}$.

If $\nabla u \cdot e \leq \ell$, then $\bar{w} \geq\left(1-e^{c_{2}(\ell-1)}\right) / c_{2}$. Therefore, it follows from the assumption that

$$
\left|\left\{(x, t) \in Q_{1}: \bar{w} \geq\left(1-e^{c_{2}(\ell-1)}\right) / c_{2}\right\}\right|>\mu\left|Q_{1}\right| .
$$

By Proposition 2.3 in [12], there exist $\tau_{1}>0$ depending only $\mu$ and $n$, and $\nu>0$ depending only on $\mu, \ell, n, \gamma$ and $p$ such that

$$
\bar{w} \geq \nu \quad \text { in } Q_{\tau_{1}} .
$$


Meanwhile, we have

$$
\bar{w} \leq W-w .
$$

This implies that

$$
W-w \geq \nu \quad \text { in } Q_{\tau_{1}} .
$$

Therefore, we have

$$
\nabla u \cdot e+\rho|\nabla u|^{2} \leq 1+\rho-\nu \quad \text { in } Q_{\tau_{1}} .
$$

Since $|\nabla u \cdot e| \leq|\nabla u|$, we have

$$
\nabla u \cdot e+\rho(\nabla u \cdot e)^{2} \leq 1+\rho-\nu \quad \text { in } Q_{\tau_{1}} .
$$

Therefore, remarking that $\nu \leq 1+\rho$, we have

$$
\nabla u \cdot e \leq \frac{-1+\sqrt{1+4 \rho(1+\rho-\nu)}}{2 \rho} \leq 1-\delta \quad \text { in } Q_{\tau_{1}}
$$

for some $\delta>0$ depending only on $p, \gamma, \mu, \ell, n$. Finally, we can choose $\tau=\tau_{1}$ if $\gamma<0$ and $\tau=\tau_{1}(1-\delta)^{\gamma / 2}$ if $\gamma \geq 0$ such that $Q_{\tau}^{1-\delta} \subset Q_{\tau_{1}}$.

Note that our choice of $\tau$ and $\delta$ in the above implies that

$$
\tau<(1-\delta)^{\frac{\gamma}{2}} \quad \text { when } \gamma \geq 0 .
$$

In the rest of the paper, we will choose $\tau$ even smaller such that

$$
\tau<(1-\delta)^{1+\gamma} \text { for all } \gamma>-1
$$

This fact will be used in the proof of Theorem 4.8.

In case we can apply the previous lemma holds in all directions $e \in \partial B_{1}$, then it effectively implies a reduction in the oscillation of $\nabla u$ in a smaller parabolic cylinder. If such improvement of oscillation takes place at all scales, it leads to the Hölder continuity of $\nabla u$ at $(0,0)$ by iteration and scaling. The following corollary describes this favorable case in which the assumption of the previous Lemma holds in all directions.

Corollary 4.2. Let $u$ be a smooth solution of (3) with $\varepsilon \in(0,1)$ such that $|\nabla u| \leq 1$ in $Q_{1}$. For every $0<\ell<1, \mu>0$, there exist $\tau \in(0,1 / 4)$ depending only on $\mu$ and $n$, and $\delta>0$ depending only on $n, p, \gamma, \mu, \ell$, such that for every nonnegative integer $k \leq \log \varepsilon / \log (1-\delta)$, if

$$
\left|\left\{(x, t) \in Q_{\tau^{i}}^{(1-\delta)^{i}}: \nabla u \cdot e \leq \ell(1-\delta)^{i}\right\}\right|>\mu\left|Q_{\tau^{i}}^{(1-\delta)^{i}}\right| \quad \text { for all } e \in \mathbb{S}^{n-1} \text { and } i=0, \cdots, k,
$$

then

$$
|\nabla u|<(1-\delta)^{i+1} \quad \text { in } Q_{\tau^{i+1}}^{(1-\delta)^{i+1}} \text { for all } i=0, \cdots, k
$$

Remark 4.3. Remark that we can further impose on $\delta$ that $\delta<1 / 2$ and $\delta<1-\tau$. 
Proof. When $i=0$, it follows from Lemma 4.1 that $\nabla u \cdot e<1-\delta$ in $Q_{\tau}$ for all $e \in \mathbb{S}^{n-1}$. This implies that $|\nabla u|<1-\delta$ in $Q_{\tau}^{1-\delta}$.

Suppose this corollary holds for $i=0, \cdots, k-1$. We are going prove it for $i=k$. Let

$$
v(x, t)=\frac{1}{\tau^{k}(1-\delta)^{k}} u\left(\tau^{k} x, \tau^{2 k}(1-\delta)^{-k \gamma} t\right) .
$$

Then $v$ satisfies

$$
v_{t}=\left(|\nabla v|^{2}+\frac{\varepsilon^{2}}{(1-\delta)^{2 k}}\right)^{\gamma / 2}\left(\Delta v+(p-2) \frac{v_{i} v_{j}}{|\nabla v|^{2}+\varepsilon^{2}(1-\delta)^{-2 k}} v_{i j}\right) \quad \text { in } Q_{1} .
$$

By the induction hypothesis, we also know that $|\nabla v| \leq 1$ in $Q_{1}$, and

$$
\left|\left\{(x, t) \in Q_{1}: \nabla v \cdot e \leq \ell\right\}\right|>\mu\left|Q_{1}\right| \text { for all } e \in \mathbb{S}^{n-1} .
$$

Notice that $\varepsilon \leq(1-\delta)^{k}$. Therefore, by Lemma 4.1 we have

$$
\nabla v \cdot e \leq 1-\delta \quad \text { in } Q_{\tau}^{1-\delta} \text { for all } e \in \mathbb{S}^{n-1} .
$$

Hence, $|\nabla v| \leq 1-\delta$ in $Q_{\tau}^{1-\delta}$. Consequently,

$$
|\nabla u|<(1-\delta)^{k+1} \quad \text { in } Q_{\tau^{k+1}}^{(1-\delta)^{k+1}}
$$

Unless $\nabla u(0,0)=0$, the above iteration will inevitably stop at some step. There will be a first value of $k$ where the assumptions of Corollary 4.2 do not hold in some direction $e \in \mathbb{S}^{n-1}$. This means that $\nabla u$ is close to some fixed vector in a large portion of $Q_{\tau^{k}}^{(1-\delta)^{k}}$. We then prove that $u$ is close to some linear function, from which the Hölder continuity of $\nabla u$ will follow applying a result from [23].

Having $\nabla u$ close to a vector $e$ for most points tells us that for every fixed time $t$, the function $u(x, t)$ will be approximately linear. However, it does not say anything about how $u$ varies respect to time. We must use the equation in order to prove that the function $u(x, t)$ will be close to some linear function uniformly in $t$. That is the main purpose of the following set of lemmas.

Lemma 4.4. Let $u \in C\left(\bar{Q}_{1}\right)$ be a smooth solution of (3) with $\gamma>-1, \varepsilon \in(0,1)$ such that $|\nabla u| \leq M$ in $Q_{1}$. Let $A$ be a positive constant. Assume that for all $t \in[-1,0]$, we have

$$
\operatorname{osc}_{B_{1}} u(\cdot, t) \leq A
$$

then

$$
\operatorname{osc}_{Q_{1}} u \leq\left\{\begin{array}{l}
C A, \quad \text { if } \gamma \geq 0 \\
C\left(A+A^{1+\gamma}\right) \quad \text { if }-1<\gamma<0,
\end{array}\right.
$$

where $C$ is a positive constant depending only on $M, \gamma, p$ and the dimension $n$.

Proof. When $\gamma \geq 0$, for the $a_{i j}$ in (24), we have $\left|a_{i j}\right| \leq \Lambda:=\left(M^{2}+1\right)^{\gamma / 2} \max (p-1,1)$, and therefore, the conclusion follows from the same proof of Lemma 4.3 in [12]. 
When $\gamma \in(-1,0)$, we choose different comparison functions from [12]. Let

$$
\begin{aligned}
& \bar{w}(x, t)=\bar{a}+\Lambda A^{1+\gamma} t+2 A|x|^{\beta}, \\
& \underline{w}(x, t)=\underline{a}-\Lambda A^{1+\gamma} t-2 A|x|^{\beta}
\end{aligned}
$$

where $\beta=\frac{2+\gamma}{1+\gamma}$ and $\Lambda$ to be fixed later. As far as $\bar{a}$ and $\underline{a}$ are concerned, $\bar{a}$ is chosen so that $\bar{w}(\cdot,-1) \geq u(\cdot,-1)$ in $B_{1}$ and $\bar{w}(\bar{x},-1)=u(\bar{x},-1)$ for some $\bar{x} \in \bar{B}_{1}$, and $\underline{a}$ is chosen so that $\underline{w}(\cdot,-1) \leq u(\cdot,-1)$ in $B_{1}$ and $\underline{w}(\underline{x},-1)=u(\underline{x},-1)$ for some $\underline{x} \in \bar{B}_{1}$. This implies that

$$
\bar{a}-\underline{a}=u(\bar{x},-1)-u(\underline{x},-1)+2 \Lambda A^{1+\gamma}-2 A|\bar{x}|^{2}-2 A|\underline{x}|^{2} \leq A+2 \Lambda A^{1+\gamma} .
$$

Notice that $\beta>2$ since $\gamma \in(-1,0)$. We now remark that if $\Lambda$ is chosen as follows: $\Lambda=$ $(2 \beta)^{\gamma+1}(\beta-1) p n^{2}+1$ then the following first inequality

$$
\Lambda A^{1+\gamma} \leq\left(\left(2 A \beta|x|^{\beta-1}\right)^{2}+\varepsilon^{2}\right)^{\gamma / 2} \cdot p n^{2} \cdot 2 A \beta(\beta-1)|x|^{\beta-2} \leq(2 \beta)^{\gamma+1}(\beta-1) p n^{2} A^{1+\gamma},
$$

(we used that $\gamma<0$ ) cannot hold true for $x \in B_{1}$. This implies that $\bar{w}$ is a strict super-solution of the equation satisfied by $u$. Similarly, $\underline{w}$ is a strict sub-solution.

We claim that

$$
\bar{w} \geq u \quad \text { in } Q_{1} \quad \text { and } \underline{w} \leq u \quad \text { in } Q_{1} .
$$

We only justify the first inequality since we can proceed similarly to get the second one. If not, let $m=-\inf _{Q_{1}}(\bar{w}-u)>0$ and $\left(x_{0}, t_{0}\right) \in \bar{Q}_{1}$ be such that $m=u\left(x_{0}, t_{0}\right)-\bar{w}\left(x_{0}, t_{0}\right)$. Then $\bar{w}+m \geq u$ in $Q_{1}$ and $\bar{w}\left(x_{0}, t_{0}\right)+m=u\left(x_{0}, t_{0}\right)$. By the choice of $\bar{a}$, we know that $t_{0}>-1$. If $x_{0} \in \partial B_{1}$, then

$$
2 A=\left(\bar{w}\left(x_{0}, t_{0}\right)+m\right)-\left(\bar{w}\left(0, t_{0}\right)+m\right) \leq u\left(x_{0}, t_{0}\right)-u\left(0, t_{0}\right) \leq \operatorname{osc}_{B_{1}} u\left(\cdot, t_{0}\right) \leq A,
$$

which is impossible. Therefore, $x_{0} \in B_{1}$. But this is not possible since $\bar{w}$ is a strict super-solution of the equation satisfied by $u$. This proves the claim.

Therefore, we have

$$
\operatorname{osc}_{Q_{1}} u \leq \sup _{Q_{1}} \bar{w}-\inf _{Q_{1}} \underline{w} \leq \bar{a}-\underline{a}+4 A=2 \Lambda A^{\gamma+1}+5 A .
$$

Lemma 4.5. Let $u \in C\left(\bar{Q}_{1}\right)$ be a smooth solution of (3) with $\gamma \in \mathbb{R}, \varepsilon \in(0,1)$. Let e $\in \mathbb{S}^{n-1}$ and $0<\delta<1 / 8$. Assume that for all $t \in[-1,0]$, we have

$$
\operatorname{osc}_{x \in B_{1}}(u(x, t)-x \cdot e) \leq \delta
$$

then

$$
\operatorname{Osc}_{(x, t) \in Q_{1}}(u(x, t)-x \cdot e) \leq C \delta,
$$

where $C$ is a positive constant depending only on $\gamma, p$ and the dimension $n$.

Proof. Let

$$
\begin{aligned}
& \bar{w}(x, t)=\bar{a}+x \cdot e+\Lambda \delta t+2 \delta|x|^{2}, \\
& \underline{w}(x, t)=\underline{a}+x \cdot e-\Lambda \delta t-2 \delta|x|^{2},
\end{aligned}
$$


where $\Lambda>0$ will be fixed later, $\bar{a}$ is chosen so that $\bar{w}(\cdot,-1) \geq u(\cdot,-1)$ in $B_{1}$ and $\bar{w}(\bar{x},-1)=$ $u(\bar{x},-1)$ for some $\bar{x} \in \bar{B}_{1}$, and $\underline{a}$ is chosen so that $\underline{w}(\cdot,-1) \leq u(\cdot,-1)$ in $B_{1}$ and $\underline{w}(\underline{x},-1)=$ $u(\underline{x},-1)$ for some $\underline{x} \in \bar{B}_{1}$. This implies that

$$
\bar{a}-\underline{a}=u(\bar{x},-1)-\bar{x} \cdot e-(u(\underline{x},-1)-\underline{x} \cdot e)+2 \Lambda \delta-2 \delta|\bar{x}|^{2}-2 \delta|\underline{x}|^{2} \leq(2 \Lambda+1) \delta .
$$

For every $x \in \bar{B}_{1}$, and $t \in[-1,0]$, since $\delta<1 / 8$, we have

$$
|\nabla \bar{w}(x, t)| \geq|e|-4 \delta|x| \geq 1 / 2, \quad|\nabla \underline{w}(x, t)| \geq|e|-4 \delta|x| \geq 1 / 2 .
$$

Similarly, $|\nabla \bar{w}(x, t)| \leq 3 / 2$ and $|\nabla \underline{w}(x, t)| \leq 3 / 2$. Therefore, using the notation (24), there is a constant $A_{0}$ (depending on $p$ and $\gamma$ ) so that

$$
a_{i j}(\nabla \bar{w}(x, t)) \leq A_{0} \mathrm{I} \quad \text { and } \quad a_{i j}(\nabla \underline{w}(x, t)) \leq A_{0} \mathrm{I} .
$$

We choose $\Lambda=5 n A_{0}$. We claim that

$$
\bar{w} \geq u \quad \text { in } Q_{1} \quad \text { and } \underline{w} \leq u \quad \text { in } Q_{1} .
$$

We only justify the first inequality since we can proceed similarly to get the second one. If not, let $m=-\inf _{Q_{1}}(\bar{w}-u)>0$ and $\left(x_{0}, t_{0}\right) \in \bar{Q}_{1}$ be such that $m=u\left(x_{0}, t_{0}\right)-\bar{w}\left(x_{0}, t_{0}\right)$. Then $\bar{w}+m \geq u$ in $Q_{1}$ and $\bar{w}\left(x_{0}, t_{0}\right)+m=u\left(x_{0}, t_{0}\right)$. By the choice of $\bar{a}$, we know that $t_{0}>-1$. If $x_{0} \in \partial B_{1}$, then

$$
\begin{aligned}
2 \delta & =\left(\bar{w}\left(x_{0}, t_{0}\right)+m\right)-x_{0} \cdot e-\left(\bar{w}\left(0, t_{0}\right)+m\right) \\
& \leq u\left(x_{0}, t_{0}\right)-x_{0} \cdot e-u\left(0, t_{0}\right) \leq \operatorname{osc}_{x \in B_{1}}\left(u\left(x, t_{0}\right)-x \cdot e\right) \leq \delta,
\end{aligned}
$$

which is impossible. Hence, $x_{0} \in B_{1}$. Therefore, we have the classical relations:

$$
\begin{aligned}
u\left(x_{0}, t_{0}\right) & =\bar{w}\left(x_{0}, t_{0}\right)+m, \\
\nabla u\left(x_{0}, t_{0}\right) & =\nabla \bar{w}\left(x_{0}, t_{0}\right) \in \bar{B}_{3 / 2} \backslash B_{1 / 2}, \\
D^{2} u\left(x_{0}, t_{0}\right) & \leq D^{2} \bar{w}\left(x_{0}, t_{0}\right)=4 \delta \mathrm{I}, \\
\partial_{t} u\left(x_{0}, t_{0}\right) & \geq \partial_{t} \bar{w}\left(x_{0}, t_{0}\right)=\Lambda \delta .
\end{aligned}
$$

It follows that

$$
u_{t}\left(x_{0}, t_{0}\right)-a_{i j}\left(\nabla u\left(x_{0}, t_{0}\right)\right) \partial_{i j} u\left(x_{0}, t_{0}\right) \geq \bar{w}_{t}\left(x_{0}, t_{0}\right)-a_{i j}\left(\nabla \bar{w}\left(x_{0}, t_{0}\right)\right) \partial_{i j} \bar{w}\left(x_{0}, t_{0}\right)>0,
$$

which is a contradiction. This proves the claim.

Therefore, we have

$$
\operatorname{osc}_{(x, t) \in Q_{1}}(u(x, t)-x \cdot e) \leq \sup _{Q_{1}}(\bar{w}-x \cdot e)-\inf _{Q_{1}}(\underline{w}-x \cdot e) \leq \bar{a}-\underline{a}+4 \delta=(2 \Lambda+5) A .
$$

Lemma 4.6. Let $\eta$ be a positive constant and $u$ be a smooth solution of (3) with $\gamma>-1, \varepsilon \in(0,1)$ such that $|\nabla u| \leq 1$ in $Q_{1}$. Assume

$$
\left|\left\{(x, t) \in Q_{1}:|\nabla u-e|>\varepsilon_{0}\right\}\right| \leq \varepsilon_{1}
$$

for some $e \in \mathbb{S}^{n-1}$ and two positive constants $\varepsilon_{0}, \varepsilon_{1}$. Then, if $\varepsilon_{0}$ and $\varepsilon_{1}$ are sufficiently small, there exists a constant $a \in \mathbb{R}$, such that

$$
|u(x, t)-a-e \cdot x| \leq \eta \quad \text { for all }(x, t) \in Q_{1 / 2} .
$$

Here, both $\varepsilon_{0}$ and $\varepsilon_{2}$ depend only on $n, p, \gamma$ and $\eta$. 
Proof. Let $f(t):=\left|\left\{x \in B_{1}:|\nabla u(x, t)-e|>\varepsilon_{0}\right\}\right|$. By the assumptions and Fubini's theorem, we have that $\int_{-1}^{0} f(t) d t \leq \varepsilon_{1}$. It follows that for $E:=\left\{t \in(-1,0): f(t) \geq \sqrt{\varepsilon_{1}}\right\}$, we obtain

$$
|E| \leq \frac{1}{\sqrt{\varepsilon_{1}}} \int_{E} f(t) d t \leq \frac{1}{\sqrt{\varepsilon_{1}}} \int_{-1}^{0} f(t) d t \leq \sqrt{\varepsilon_{1}} .
$$

Therefore, for all $t \in(-1,0] \backslash E$, with $|E| \leq \sqrt{\varepsilon_{1}}$, we have

$$
\left|\left\{x \in B_{1}:|\nabla u(x, t)-e|>\varepsilon_{0}\right\}\right| \leq \sqrt{\varepsilon_{1}} .
$$

It follows from (27) and Morrey's inequality that for all $t \in(-1,0] \backslash E$, we have

$$
\operatorname{osc}_{B_{1 / 2}}(u(\cdot, t)-e \cdot x) \leq C(n)\|\nabla u-e\|_{L^{2 n}\left(B_{1}\right)} \leq C(n)\left(\varepsilon_{0}+\varepsilon_{1}^{\frac{1}{4 n}}\right),
$$

where $C(n)>0$ depends only on $n$.

Meanwhile, since $|\nabla u| \leq 1$ in $Q_{1}$, we have that $\operatorname{osc}_{B_{1}} u(\cdot, t) \leq 2$ for all $t \in(-1,0]$. Thus, applying Lemma 4.4, we have that $\operatorname{osc}_{Q_{1}} u \leq C$ for some constant $C$. Note that $u(t, x)-u(0,0)$ also satisfies (3) and $\|u(t, x)-u(0,0)\|_{L^{\infty}\left(Q_{1}\right)} \leq \operatorname{osc}_{Q_{1}} u \leq C$. By applying Lemma 3.1 to $u(t, x)-u(0,0)$, we have

$$
\sup _{t \neq s,(t, x),(s, x) \in Q_{1}} \frac{|u(t, x)-u(s, x)|}{|t-s|^{1 / 2}} \leq C .
$$

Therefore, by (28) and the fact that $|E| \leq \sqrt{\varepsilon_{1}}$, we obtain

$$
\operatorname{Osc}_{B_{1 / 2}}(u(\cdot, t)-e \cdot x) \leq C\left(\varepsilon_{0}+\varepsilon_{1}^{\frac{1}{4 n}}+\varepsilon_{1}^{\frac{1}{4}}\right)
$$

for all $t \in(-1 / 4,0]$ (that is, including $t \in E$ ). If $\varepsilon_{0}$ and $\varepsilon_{1}$ are sufficiently small, we obtain from Lemma 4.5 that

$$
\operatorname{osc}_{Q_{1 / 2}}(u-e \cdot x) \leq C\left(\varepsilon_{0}+\varepsilon_{1}^{\frac{1}{4 n}}+\varepsilon_{1}^{\frac{1}{4}}\right) .
$$

Hence, if $\varepsilon_{0}$ and $\varepsilon_{1}$ are sufficiently small, there exists a constant $a \in \mathbb{R}$, such that

$$
|u(t, x)-a-e \cdot x| \leq \eta \quad \text { for all }(x, t) \in Q_{1 / 2} .
$$

Theorem 4.7 (Regularity of small perturbation solutions). Let $u$ be a smooth solution of (3) in $Q_{1}$. For each $\beta \in(0,1)$, there exist two positive constants $\eta$ (small) and $C$ (large), both of which depends only on $\beta, n, \gamma$ and $p$, such that if $|u(x, t)-L(x)| \leq \eta$ in $Q_{1}$ for some linear function $L$ of $x$ satisfying $1 / 2 \leq|\nabla L| \leq 2$, then

$$
\|u-L\|_{C^{2, \beta}\left(Q_{1 / 2}\right)} \leq C
$$

Proof. Since $L$ is a solution of (3), the conclusion follows from Corollary 1.2 in [23].

Now we are ready to prove the following Hölder gradient estimate. 
Theorem 4.8. Let $u$ be a smooth solution of (3) with $\varepsilon \in(0,1), \gamma>-1$ such that $|\nabla u| \leq 1$ in $Q_{1}$. Then there exist two positive constants $\alpha$ and $C$ depending only on $n, \gamma$ and $p$ such that

$$
|\nabla u(x, t)-\nabla u(y, s)| \leq C\left(|x-y|^{\alpha}+|t-s|^{\frac{\alpha}{2-\alpha \gamma}}\right)
$$

for all $(x, t),(y, s) \in Q_{1 / 2}$. Also, there holds

$$
|u(x, t)-u(x, s)| \leq C|t-s|^{\frac{1+\alpha}{2-\alpha \gamma}}
$$

for all $(x, t),(x, s) \in Q_{1 / 2}$.

Proof. We first show the Hölder estimate of $\nabla u$ at $(0,0)$ and the Hölder estimate in $t$ at $(0,0)$.

Let $\eta$ be the one in Theorem 4.7 with $\beta=1 / 2$, and for this $\eta$, let $\varepsilon_{0}, \varepsilon_{1}$ be two sufficiently small positive constants so that the conclusion of Lemma 4.6 holds. For $\ell=1-\varepsilon_{0}^{2} / 2$ and $\mu=\varepsilon_{1} /\left|Q_{1}\right|$, if

$$
\left|\left\{(x, t) \in Q_{1}: \nabla u \cdot e \leq \ell\right\}\right| \leq \mu\left|Q_{1}\right| \text { for any } e \in \mathbb{S}^{n-1},
$$

then

$$
\left|\left\{(x, t) \in Q_{1}:|\nabla u-e|>\varepsilon_{0}\right\}\right| \leq \varepsilon_{1} .
$$

This is because if $|\nabla u(x, t)-e|>\varepsilon_{0}$ for some $(x, t) \in Q_{1}$, then

$$
|\nabla u|^{2}-2 \nabla u \cdot e+1 \geq \varepsilon_{0}^{2}
$$

Since $|\nabla u| \leq 1$, we have

$$
\nabla u \cdot e \leq 1-\varepsilon_{0}^{2} / 2 .
$$

Therefore, if $\ell=1-\varepsilon_{0}^{2} / 2$ and $\mu=\varepsilon_{1} /\left|Q_{1}\right|$, then

$$
\left\{(x, t) \in Q_{1}:|\nabla u-e|>\varepsilon_{0}\right\} \subset\left\{(x, t) \in Q_{1}: \nabla u \cdot e \leq \ell\right\},
$$

from which it follows that

$$
\left|\left\{(x, t) \in Q_{1}:|\nabla u-e|>\varepsilon_{0}\right\}\right| \leq\left|\left\{(x, t) \in Q_{1}: \nabla u \cdot e \leq \ell\right\}\right| \leq \mu\left|Q_{1}\right| \leq \varepsilon_{1} .
$$

Let $\tau, \delta$ be the constants in Corollary 4.2. Denote $[\log \varepsilon / \log (1-\delta)]$ as the integer part of $\log \varepsilon / \log (1-\delta)$. Let $k$ be either $[\log \varepsilon / \log (1-\delta)]$ or the minimum nonnegative integer such that the condition (26) does not hold, whichever is smaller. Then it follows from Corollary 4.2 that for all $\ell=0,1, \cdots, k$, we have

$$
|\nabla u(x, t)| \leq(1-\delta)^{\ell} \quad \text { in } Q_{\tau^{\ell}}^{(1-\delta)^{\ell}} .
$$

Then for $(x, t) \in Q_{\tau^{\ell}}^{(1-\delta)^{\ell}} \backslash Q_{\tau^{\ell+1}}^{(1-\delta)^{\ell+1}}$,

$$
|\nabla u(x, t)| \leq(1-\delta)^{\ell} \leq C\left(|x|^{\alpha}+|t|^{\frac{\alpha}{2-\alpha \gamma}}\right),
$$

where $C=\frac{1}{1-\delta}$ and $\alpha=\frac{\log (1-\delta)}{\log \tau}$. Thus,

$$
|\nabla u(x, t)-q| \leq C\left(|x|^{\alpha}+|t|^{\frac{\alpha}{2-\alpha \gamma}}\right) \quad \text { in } Q_{1} \backslash Q_{\tau^{k+1}}^{(1-\delta)^{k+1}}
$$


for every $q \in \mathbb{R}^{n}$ such that $|q| \leq(1-\delta)^{k}$. Note that when $\gamma \geq 0$, it follows from (25) that

$$
2-\alpha \gamma>0 \text { and } \frac{\alpha}{2-\alpha \gamma}<\frac{1}{2} .
$$

For $\ell=0,1, \cdots, k$, let

$$
u_{\ell}(x, t)=\frac{1}{\tau^{\ell}(1-\delta)^{\ell}} u\left(\tau^{\ell} x, \tau^{2 \ell}(1-\delta)^{-\ell \gamma} t\right) .
$$

Then $\left|\nabla u_{\ell}(x, t)\right| \leq 1$ in $Q_{1}$, and

$$
\partial_{t} u_{\ell}=\left(\left|\nabla u_{\ell}\right|^{2}+\varepsilon^{2}(1-\delta)^{-2 \ell}\right)^{\gamma / 2}\left(\delta_{i j}+(p-2) \frac{\partial_{i} u_{\ell} \partial_{j} u_{\ell}}{\left|\nabla u_{\ell}\right|^{2}+\varepsilon^{2}(1-\delta)^{-2 \ell}}\right) \partial_{i j} u_{\ell} \quad \text { in } Q_{1} .
$$

Notice that $\varepsilon^{2}(1-\delta)^{-2 \ell} \leq \varepsilon^{2}(1-\delta)^{-2 k} \leq 1$. By Lemma 4.4, we have

$$
\operatorname{osc}_{Q_{1}} u_{\ell} \leq C,
$$

and thus,

$$
\operatorname{osc}_{Q_{\tau \ell}^{(1-\delta)^{\ell}}} u \leq C \tau^{\ell}(1-\delta)^{\ell}
$$

Let $v=u_{k}$.

Case 1: $k=[\log \varepsilon / \log (1-\delta)]$. Then we have $(1-\delta)^{k+1}<\varepsilon \leq(1-\delta)^{k}$, and thus, $\frac{1}{2}<1-\delta<\varepsilon(1-\delta)^{-k} \leq 1$. Therefore, when $\ell=k$, the equation (34) is a uniformly parabolic quasilinear equation with smooth and bounded coefficients. By the standard quasilinear parabolic equation theory (see, e.g., Theorem 4.4 of [15] in page 560) and Schauder estimates, there exists $b \in \mathbb{R}^{n},|b| \leq 1$ such that

$$
|\nabla v(x, t)-b| \leq C\left(|x|+|t|^{1 / 2}\right) \leq C\left(|x|^{\alpha}+|t|^{\frac{\alpha}{2-\alpha \gamma}}\right) \quad \text { in } Q_{\tau}^{1-\delta} \subset Q_{1 / 4}
$$

and

$$
\left|\partial_{t} v\right| \leq C \quad \text { in } Q_{\tau}^{1-\delta} \subset Q_{1 / 4},
$$

where $C>0$ depends only on $\gamma, p$ and $n$, and we used that $\frac{\alpha}{2-\alpha \gamma} \leq \frac{1}{2}$. Rescaling back, we have

$$
\left|\nabla u(x, t)-(1-\delta)^{k} b\right| \leq C\left(|x|^{\alpha}+|t|^{\frac{\alpha}{2-\alpha \gamma}}\right) \quad \text { in } Q_{\tau^{k+1}}^{(1-\delta)^{k+1}}
$$

and

$$
|u(x, t)-u(x, 0)| \leq C \tau^{-k}(1-\delta)^{k(\gamma+1)}|t| \quad \text { in } Q_{\tau^{k+1}}^{(1-\delta)^{k+1}} .
$$

Then we can conclude from (31) and (36) that

$$
|\nabla u(x, t)-q| \leq C\left(|x|^{\alpha}+|t|^{\frac{\alpha}{2-\alpha \gamma}}\right) \quad \text { in } Q_{1 / 2}
$$

where $C>0$ depends only on $\gamma, p$ and $n$. From (37), we obtain that for $|t| \leq \tau^{2 m}(1-\delta)^{-m \gamma}$ with $m \geq k+1$,

$$
|u(0, t)-u(0,0)| \leq C \tau^{-k}(1-\delta)^{k(\gamma+1)} \tau^{2 m}(1-\delta)^{-m \gamma} \leq C \tau^{m}(1-\delta)^{m},
$$


where in the last inequality we have used (25). From (35) and (38), we have

$$
|u(0, t)-u(0,0)| \leq C|t|^{\beta}
$$

for all $t \in(-1 / 4,0]$, where $\beta$ is chosen such that

$$
\tau(1-\delta)=\left(\tau^{2}(1-\delta)^{-\gamma}\right)^{\beta} .
$$

That is,

$$
\beta=\frac{1+\alpha}{2-\alpha \gamma}
$$

Note that $\beta>\frac{1}{2}$ if $\gamma>-2$.

Case 2: $k<[\log \varepsilon / \log (1-\delta)]$. Then

$$
\left|\left\{(x, t) \in Q_{\tau^{k}}^{(1-\delta)^{k}}: \nabla u \cdot e \leq \ell(1-\delta)^{k}\right\}\right| \leq \mu\left|Q_{\tau^{k}}^{(1-\delta)^{k}}\right| \quad \text { for some } e \in \mathbb{S}^{n-1} .
$$

Also,

$$
|\nabla u|<(1-\delta)^{\ell} \quad \text { in } Q_{\tau^{\ell}}^{(1-\delta)^{\ell}} \text { for all } \ell=0,1, \cdots, k .
$$

Recall $v=u_{k}$ as defined in (33), which satisfies (34) with $\ell=k$. Then $|\nabla v| \leq 1$ in $Q_{1}$, and

$$
\left|\left\{(x, t) \in Q_{1}: \nabla v \cdot e \leq \ell\right\}\right| \leq \mu\left|Q_{1}\right| \text { for some } e \in \mathbb{S}^{n-1} .
$$

Consequently, using (29), we get

$$
\left|\left\{(x, t) \in Q_{1}:|\nabla v-e|>\varepsilon_{0}\right\}\right| \leq \varepsilon_{1} .
$$

It follows from Lemma 4.6 that there exists $a \in \mathbb{R}$ such that

$$
|v(x, t)-a-e \cdot x| \leq \eta \quad \text { for all }(x, t) \in Q_{1 / 2} .
$$

By Theorem 4.7, there exists $b \in \mathbb{R}^{n}$ such that

$$
|\nabla v-b| \leq C(|x|+\sqrt{|t|}) \quad \text { for all }(x, t) \in Q_{\tau}^{1-\delta} \subset Q_{1 / 4} .
$$

and

$$
\left|\partial_{t} v\right| \leq C \quad \text { in } Q_{\tau}^{1-\delta} \subset Q_{1 / 4}
$$

Rescaling back, we have

$$
\left|\nabla u(x, t)-(1-\delta)^{k} b\right| \leq C\left(|x|^{\alpha}+|t|^{\frac{\alpha}{2-\alpha \gamma}}\right) \quad \text { in } Q_{\tau^{k+1}}^{(1-\delta)^{k+1}}
$$

and

$$
|u(x, t)-u(x, 0)| \leq C \tau^{-k}(1-\delta)^{k(\gamma+1)}|t| \quad \text { in } Q_{\tau^{k+1}}^{(1-\delta)^{k+1}} .
$$

Together with (31) and (35), we can conclude as in Case 1 that

$$
|\nabla u(x, t)-q| \leq C\left(|x|^{\alpha}+|t|^{\frac{\alpha}{2-\alpha \gamma}}\right) \quad \text { in } Q_{1 / 2}
$$


and

$$
|u(0, t)-u(0,0)| \leq C|t|^{\beta},
$$

for all $t \in(-1 / 4,0]$, where $C>0$ depends only on $\gamma, p$ and $n$.

In conclusion, we have proved that there exist $q \in \mathbb{R}^{n}$ with $|q| \leq 1$, and two positive constants $\alpha, C$ depending only on $\gamma, p$ and $n$ such that

$$
|\nabla u(x, t)-q| \leq C\left(|x|^{\alpha}+|t|^{\frac{\alpha}{2-\alpha \gamma}}\right) \quad \text { for all }(x, t) \in Q_{1 / 2}
$$

and

$$
|u(0, t)-u(0,0)| \leq C|t|^{\beta}, \quad \text { for } t \in(-1 / 4,0],
$$

where $\beta$ is given in (39). Then the conclusion follows from standard translation arguments.

\section{Approximation}

As mentioned in the introduction, the viscosity solutions to

$$
u_{t}=|\nabla u|^{\gamma}\left(\Delta u+(p-2)|\nabla u|^{-2} u_{i} u_{j} u_{i j}\right) \quad \text { in } Q_{1}
$$

with $\gamma>-1$ and $p>1$ fall into the general framework studied by Ohnuma-Sato in [19], which is an extension of the work of Barles-Georgelin [5] and Ishii-Souganidis [11] on the viscosity solutions of singular/degenerate parabolic equations. Let us recall the definition of viscosity solutions to (40) in [19].

We denote

$$
F\left(\nabla u, \nabla^{2} u\right)=|\nabla u|^{\gamma}\left(\Delta u+(p-2)|\nabla u|^{-2} u_{i} u_{j} u_{i j}\right) .
$$

Let $\mathcal{F}$ be the set of functions $f \in C^{2}([0, \infty))$ satisfying

$$
f(0)=f^{\prime}(0)=f^{\prime \prime}(0)=0, \quad f^{\prime \prime}(r)>0 \text { for all } r>0,
$$

and

$$
\lim _{|x| \rightarrow 0, x \neq 0} F\left(\nabla g(x), \nabla^{2} g(x)\right)=\lim _{|x| \rightarrow 0, x \neq 0} F\left(-\nabla g(x),-\nabla^{2} g(x)\right)=0, \quad \text { where } g(x)=f(|x|) .
$$

This set $\mathcal{F}$ is not empty when $\gamma>-1$ and $p>1$, since $f(r)=r^{\beta} \in \mathcal{F}$ for any $\beta>\max \left(\frac{\gamma+2}{\gamma+1}, 2\right)$. Moreover, if $f \in \mathcal{F}$, then $\lambda f \in \mathcal{F}$ for all $\lambda>0$.

Because the equation (40) may be singular or degenerate, one needs to choose the test functions properly when defining viscosity solutions. A function $\varphi \in C^{2}\left(Q_{1}\right)$ is admissible, which is denoted as $\varphi \in \mathcal{A}$, if for every $\hat{z}=(\hat{z}, \hat{t}) \in Q_{1}$ that $\nabla \varphi(\hat{z})=0$, there exist $\delta>0, f \in \mathcal{F}$ and $\omega \in C([0, \infty))$ satisfying $\omega \geq 0$ and $\lim _{r \rightarrow 0} \frac{\omega(r)}{r}=0$ such that for all $z=(x, t) \in Q_{1},|z-\hat{z}|<\delta$ we have

$$
\left|\varphi(z)-\varphi(\hat{z})-\varphi_{t}(\hat{z})(t-\hat{t})\right| \leq f(|x-\hat{x}|)+\omega(|t-\hat{t}|) .
$$


Definition 5.1. An upper (lower, resp.) semi-continuous function $u$ in $Q_{1}$ is called a viscosity subsolution (supersolution, resp.) of (40) if for every $\varphi \in C^{2}\left(Q_{1}\right), u-\varphi$ has a local maximum (minimum, resp.) at $\left(x_{0}, t_{0}\right) \in Q_{1}$, then

$$
\varphi_{t} \leq(\geq \text {,resp. })|\nabla \varphi|^{\gamma}\left(\Delta \varphi+(p-2)|\nabla \varphi|^{-2} \varphi_{i} \varphi_{j} \varphi_{i j}\right) \text { at }\left(x_{0}, t_{0}\right) \text { when } \nabla \varphi\left(x_{0}, t_{0}\right) \neq 0
$$

and

$$
\varphi_{t} \leq(\geq \text {,resp. }) 0 \text { at }\left(x_{0}, t_{0}\right) \text { when } \nabla \varphi\left(x_{0}, t_{0}\right)=0 .
$$

A function $u \in C\left(Q_{1}\right)$ is called a viscosity solution of (1), if it is both a viscosity subsolution and a viscosity supersolution.

We shall use two properties about the viscosity solutions defined in the above. The first one is the comparison principle for (40), which is Theorem 3.1 in [19].

Theorem 5.2 (Comparison principle). Let $u$ and $v$ be a viscosity subsolution and a viscosity supersolution of (40) in $Q_{1}$, respectively. If $u \leq v$ on $\partial_{p} Q_{1}$, then $u \leq v$ in $\bar{Q}_{1}$.

The second one is the stability of viscosity solutions of (40), which is an application of Theorem 6.1 in [19]. Its application to the equation (40) with $\gamma=0,1<p \leq 2$ is given in Proposition 6.2 in [19] with detailed proof. It is elementary to check it applies to (40) for all $\gamma>-1$ and all $p>1$ (which was also pointed out in [19]).

Theorem 5.3 (Stability). Let $\left\{u_{k}\right\}$ be a sequence of bounded viscosity subsolutions of (3) in $Q_{1}$ with $\varepsilon_{k} \geq 0$ that $\varepsilon_{k} \rightarrow 0$, and $u_{k}$ converges locally uniformly to $u$ in $Q_{1}$. Then $u$ is a viscosity subsolution of (40) in $Q_{1}$.

Now we shall use the solution of (3) to approximate the solution of (40). Since $p>1$, it follows from classical quasilinear equations theory (see e.g. [15, Theorem 4.4, p. 560]) and the Schauder estimates that

Lemma 5.4. Let $g \in C\left(\partial_{p} Q_{1}\right)$. For $\varepsilon>0$, there exists a unique solution $u^{\varepsilon} \in C^{\infty}\left(Q_{1}\right) \cap C\left(\bar{Q}_{1}\right)$ of (3) with $p>1$ and $\gamma \in \mathbb{R}$ such that $u^{\varepsilon}=g$ on $\partial_{p} Q_{1}$.

The last ingredient we need in the proof of Theorem 1.1 is the following continuity estimate up to the boundary for the solutions of (3), where the proof is given in the appendix. For two real numbers $a$ and $b$, we denote $a \vee b=\max (a, b), a \wedge b=\min (a, b)$.

Theorem 5.5 (Boundary estimates). Let $u \in C\left(\bar{Q}_{1}\right) \cap C^{\infty}\left(Q_{1}\right)$ be a solution of (3) with $\gamma>-1$ and $\varepsilon \in(0,1)$. Let $\varphi:=\left.u\right|_{\partial_{p} Q_{1}}$ and let $\rho$ be a modulus of continuity of $\varphi$. Then there exists another modulus of continuity $\rho^{*}$ depending only on $n, \gamma, p, \rho,\|\varphi\|_{L^{\infty}\left(\partial_{p} Q_{1}\right)}$ such that

$$
|u(x, t)-u(y, s)| \leq \rho^{*}(|x-y| \vee \sqrt{|t-s|})
$$

for all $(x, t),(y, s) \in \bar{Q}_{1}$.

Proof of Theorem 1.1. Given Theorem 4.8, Theorem 5.2, Theorem 5.3, Lemma 5.4 and Theorem 5.5 , the proof of Theorem 1.1 is identical to that of Theorem 1 in [12]. 


\section{A Appendix}

We will adapt some arguments in [6] to prove Theorem 5.5. In the following, $c$ denotes some positive constant depending only on $n, \gamma$ and $p$, which may vary from line to line. Denote

$$
F_{\varepsilon}\left(\nabla u, \nabla^{2} u\right)=\left(|\nabla u|^{2}+\varepsilon^{2}\right)^{\gamma / 2}\left(\delta_{i j}+(p-2) \frac{u_{i} u_{j}}{|\nabla u|^{2}+\varepsilon^{2}}\right) u_{i j} .
$$

Lemma A.1. For every $z \in \partial B_{1}$, there exists a function $W_{z} \in C\left(\bar{B}_{1}\right)$ such that $W_{z}(z)=$ $0, W_{z}>0$ in $\bar{B}_{1} \backslash\{z\}$, and

$$
F_{\varepsilon}\left(\nabla W_{z}, \nabla^{2} W_{z}\right) \leq-1 \quad \text { in } B_{1} .
$$

Proof. Let $z \in \partial B_{1}$. Let $f(r)=\sqrt{(r-1)^{+}}$and $w_{z}(x)=f(|x-2 z|)$. Then for $x \in B_{1}$, we have

$$
F_{\varepsilon}\left(\nabla w_{z}, \nabla^{2} w_{z}\right)=\left(f^{\prime 2}+\varepsilon^{2}\right)^{\frac{\gamma}{2}}\left(\left(1+(p-2) \frac{f^{\prime 2}}{f^{\prime 2}+\varepsilon^{2}}\right) f^{\prime \prime}+\frac{n-1}{|x-2 z|} f^{\prime}\right) .
$$

Then there exists $\delta>0$ depending only on $n, \gamma$ and $p$ such that for $x \in B_{1} \cap B_{1+\delta}(2 z)$, we have

$$
F_{\varepsilon}\left(\nabla w_{z}, \nabla^{2} w_{z}\right) \leq-1 .
$$

For $\sigma=\frac{2 n}{\min (p-1,1)}+2$ and $a>0$, let $G_{z}(x)=a\left(2^{\sigma}-\frac{1}{|x-2|^{\sigma}}\right)$. Then $G_{z}(x) \geq a\left(2^{\sigma}-1\right)$ in $B_{1}$. Also, for $r=|x-2 z|$ and $x \in B_{1}$, we have

$$
\begin{aligned}
& F_{\varepsilon}\left(\nabla G_{z}, \nabla^{2} G_{z}\right) \\
& =a\left(\sigma^{2} r^{-2 \sigma-2}+\varepsilon^{2}\right)^{\frac{\gamma}{2}}\left(\left(1+\frac{(p-2) \sigma^{2}}{\sigma^{2}+\varepsilon^{2} r^{2 \sigma+2}}\right) \sigma(-\sigma-1) r^{-\sigma-2}+(n-1) \sigma r^{-\sigma-2}\right) \\
& \leq-\frac{a}{2} \sigma r^{-\sigma-2}\left(\sigma^{2} r^{-2 \sigma-2}+\varepsilon^{2}\right)^{\frac{\gamma}{2}} \\
& \leq \begin{cases}-\frac{a}{2} 3^{-\sigma-2-\gamma(\sigma+1)} \sigma^{1+\gamma} & \text { when } \gamma \geq 0 \\
-\frac{a}{2} 3^{-\sigma-2}\left(\sigma^{2}+1\right)^{\gamma / 2} \sigma & \text { when } \gamma<0,\end{cases}
\end{aligned}
$$

where in the first inequality we used the choice of $\sigma$. Then we choose $a$ that

$$
a\left(2^{\sigma}-\frac{1}{|1+\delta|^{\sigma}}\right)=\sqrt{\delta / 2}
$$

Since $w_{z}(z)=0$ and $G_{z}(z)>0$, the function

$$
W_{z}(x)=\left\{\begin{array}{l}
G_{z}(x) \quad \text { for } x \in \bar{B}_{1},|x-2 z| \geq 1+\delta \\
\min \left(G_{z}(x), w_{z}(x)\right) \quad \text { for } x \in \bar{B}_{1},|x-2 z| \leq 1+\delta
\end{array}\right.
$$

agrees with $w_{z}$ in a neighborhood of $z$ (relative to $\bar{B}_{1}$ ). Also, because of the choice of $a, W_{z}$ agrees with $G_{z}$ when $x \in \bar{B}_{1}$ and $|x-2 z| \geq 1+\tilde{\delta}$ for some $\tilde{\delta} \in(0, \delta)$. Moreover,

$$
F_{\varepsilon}\left(\nabla W_{z}, \nabla^{2} W_{z}\right) \leq-\kappa
$$

for some constant $\kappa>0$ depending only on $n, \gamma$ and $p$. By multiplying a large positive constant to $W_{z}$, we finish the proof of this lemma. 
Lemma A.2. For every $(z, \tau) \in \partial_{p} Q_{1}$, there exists $W_{z, \tau} \in C\left(\bar{Q}_{1}\right)$ such that $W_{z, \tau}(z, \tau)=0$, $W_{z, \tau}>0$ in $\bar{Q}_{1} \backslash\{(z, \tau)\}$, and

$$
\partial_{t} W_{z, \tau}-F_{\varepsilon}\left(\nabla W_{z, \tau}, \nabla^{2} W_{z, \tau}\right) \geq 1 \quad \text { in } Q_{1} .
$$

Proof. For $\tau>-1$ and $x \in \partial B_{1}$, then

$$
W_{z, \tau}(x, t)=\frac{(t-\tau)^{2}}{2}+2 W_{z}
$$

is a desired function, where $W_{z}$ is the one in Lemma A.1. For $\tau=-1$ and $x \in B_{1}$, we let

$$
W_{z, \tau}(x, t)=A(t+1)+|x-z|^{\beta},
$$

where $\beta=\max \left(\frac{\gamma+2}{\gamma+1}, 2\right)$. Then if we choose $A>0$ large, which depends only on $n, \gamma$ and $p$, then $W_{z, \tau}$ will be a desired function.

For two real numbers $a$ and $b$, we denote $a \vee b=\max (a, b), a \wedge b=\min (a, b)$.

Theorem A.3. Let $u \in C\left(\bar{Q}_{1}\right) \cap C^{\infty}\left(Q_{1}\right)$ be a solution of (3) with $\gamma>-1$ and $\varepsilon \in(0,1)$. Let $\varphi:=\left.u\right|_{\partial_{p} Q_{1}}$ and let $\rho$ be a modulus of continuity of $\varphi$. Then there exists another modulus of continuity $\rho^{*}$ depending only on $n, \gamma, p, \rho$ such that

$$
|u(x, t)-u(y, s)| \leq \tilde{\rho}(|x-y| \vee|t-s|)
$$

for all $(x, t) \in \bar{Q}_{1},(y, s) \in \partial_{p} Q_{1}$.

Proof. For every $\kappa>0$ and $(z, \tau) \in \partial_{p} Q_{1}$, let

$$
W_{\kappa, z, \tau}(x, t)=\varphi(z, \tau)+\kappa+M_{\kappa} W_{z, \tau}(x, t),
$$

where $M_{\kappa}>0$ is chose so that

$$
\varphi(z, \tau)+\kappa+M_{\kappa} W_{z, \tau}(y, s) \geq \varphi(y, s) \quad \text { for all }(y, s) \in \partial_{p} Q_{1} .
$$

Indeed,

$$
M_{k}=\inf _{(y, s) \in \partial_{p} Q_{1},(y, s) \neq(z, \tau)} \frac{(\rho(|z-y| \vee|\tau-s|)-\kappa)^{+}}{W_{z, \tau}(y, s)}
$$

would suffice, and is independent of the choice of $(z, \tau)$. Finally, let

$$
W(x, t)=\inf _{\kappa>0,(z, \tau) \in \partial_{p} Q_{1}} W_{\kappa, z, \tau}(x, t) .
$$

Note that for every $\kappa>0$ and $(z, \tau) \in \partial_{p} Q_{1}$,

$$
\begin{aligned}
W(x, t)-\varphi(z, \tau) & \leq W_{\kappa, z, \tau}(x, t)-\varphi(z, \tau) \\
& \leq \kappa+M_{\kappa} W_{z, \tau}(x, t) \\
& \leq \kappa+M_{\kappa}\left(W_{z, \tau}(x, t)-W_{z, \tau}(z, \tau)\right) \\
& \leq \kappa+M_{\kappa} \omega(|z-x| \vee|\tau-t|),
\end{aligned}
$$


where $\omega$ is the modulus of continuity for $W_{z, \tau}$, which is evidently independent of $(z, \tau)$. Let $\tilde{\rho}(r)=\inf _{\kappa>0}\left(\kappa+M_{\kappa} \omega(r)\right)$ for all $r \geq 0$. Then $\tilde{\rho}$ is a modulus of continuity, and

$$
W(x, t)-\varphi(z, \tau) \leq \tilde{\rho}(|z-x| \vee|\tau-t|) \quad \text { for all }(x, t) \in \bar{Q}_{1},(z, \tau) \in \partial_{p} Q_{1} .
$$

By Lemma A.2, $W_{\kappa, z, \tau}$ is a supersolution of (3) for every $\kappa>0$ and $(z, \tau) \in \partial_{p} Q_{1}$, and therefore, $W$ is also a supersolution of (3). By the comparison principle,

$$
u(x, t)-\varphi(z, \tau) \leq W(x, t)-\varphi(z, \tau) \leq \tilde{\rho}(|z-x| \vee|\tau-t|)
$$

for all $(x, t) \in \bar{Q}_{1},(z, \tau) \in \partial_{p} Q_{1}$.

Similarly, one can show that $u(x, t)-\varphi(z, \tau) \geq-\tilde{\rho}(|z-x| \vee|\tau-t|)$ for all $(x, t) \in$ $\bar{Q}_{1},(z, \tau) \in \partial_{p} Q_{1}$. This finishes the proof of this theorem.

Proof of Theorem 5.5. By the maximum principle, we have that

$$
M:=\|u\|_{L^{\infty}\left(Q_{1}\right)}=\|\varphi\|_{L^{\infty}\left(\partial_{p} Q_{1}\right)} .
$$

Let $(x, t),(y, s) \in Q_{1}$, and we assume that $t \geq s$. Let $x_{0}$ be such that $\left|x-x_{0}\right|=1-|x|=r$. Let $\tilde{\rho}$ be the one in the conclusion of Theorem A.3. Without loss of generality, we may assume that $2 M+2 \geq \tilde{\rho}(r) \geq r$ for all $r \in[0,2]$ (e.g., replacing $\tilde{\rho}(r)$ by $\tilde{\rho}(r)+r$ ), and $\tilde{\rho}(r) \leq 2 M+2$ for all $r \geq 2$.

In the following, if $\gamma \in(-1,0)$, then we will assume first that

$$
r^{1+\gamma}(2 M+2)^{-\gamma} \leq 1,
$$

and will deal with the other situation in the end of this proof. Under the above assumption, we have that $r^{2+\gamma}(\tilde{\rho}(2 r))^{-\gamma} \leq r^{2+\gamma}(2 M+2)^{-\gamma} \leq r$ when $\gamma<0$, and $r^{2+\gamma}(\tilde{\rho}(2 r))^{-\gamma} \leq r^{2+\gamma}(\tilde{\rho}(r))^{-\gamma} \leq$ $r^{2} \leq r$ when $\gamma \geq 0$. Thus, for all $\gamma>-1$, we have

$$
r^{2+\gamma}(\tilde{\rho}(2 r))^{-\gamma} \leq r .
$$

We will deal with the situation that $\gamma \in(-1,0)$ and $r^{1+\gamma}(2 M+2)^{-\gamma} \geq 1$ in the very end of the proof.

Case 1: $r^{2+\gamma}(\tilde{\rho}(2 r))^{-\gamma} \leq 1+t$.

If $|y-x| \leq r / 2$ and $|s-t| \leq r^{2+\gamma}(\tilde{\rho}(2 r))^{-\gamma} / 4$, then we do a scaling:

$$
v(z, \tau)=\frac{u\left(r z+x, r^{2+\gamma}(\tilde{\rho}(2 r))^{-\gamma} \tau+t\right)-u\left(x_{0}, t\right)}{\tilde{\rho}(2 r)} .
$$

Then

$$
v_{\tau}=\left(|\nabla v|^{2}+\varepsilon^{2} r^{2} \tilde{\rho}(2 r)^{-2}\right)^{\gamma / 2}\left(\delta_{i j}+(p-2) \frac{v_{i} v_{j}}{|\nabla u|^{2}+\varepsilon^{2} r^{2} \tilde{\rho}(2 r)^{-2}}\right) u_{i j} \quad \text { in } Q_{1} .
$$

Notice that $\varepsilon r / \tilde{\rho}(2 r) \leq \varepsilon r / \tilde{\rho}(r) \leq \varepsilon<1$ and $r^{2+\gamma}(\tilde{\rho}(2 r))^{-\gamma} \leq r$. Thus, $|v(z, \tau)| \leq 1$ for $(z, \tau) \in Q_{1}$. Applying Corollary 2.4 and Lemma 3.1 to $v$ and rescaling to $u$, there exists $\alpha>0$ 
depending only on $\gamma$ such that $v$ is $C^{\alpha}$ in $(x, t)$, and there exists $C>0$ depending only on $n, \gamma$ and $p$, such that

$$
|u(y, s)-u(x, s)| \leq C \tilde{\rho}(2 r) \frac{|x-y|^{\alpha}}{r^{\alpha}}
$$

and

$$
|u(x, t)-u(x, s)| \leq C \tilde{\rho}(2 r)^{1+\alpha \gamma} \frac{|t-s|^{\alpha}}{r^{\alpha(2+\gamma)}},
$$

Therefore,

$$
|u(y, s)-u(x, t)| \leq C \tilde{\rho}(2 r) \frac{|x-y|^{\alpha}}{r^{\alpha}}+C \tilde{\rho}(2 r)^{1+\alpha \gamma} \frac{|t-s|^{\alpha}}{r^{\alpha(2+\gamma)}} .
$$

Since $|y-x| \leq r / 2$ and $|s-t| \leq r^{2+\gamma}(\tilde{\rho}(2 r))^{-\gamma} / 4 \leq r / 4$, we have $2^{-m-1} r<|x-y| \vee|t-s| \leq$ $2^{-m} r$ for some integer $m \geq 1$. Then

$$
\begin{aligned}
|u(y, s)-u(x, t)| & \leq C \frac{\tilde{\rho}\left(2^{m+2}(|x-y| \vee|t-s|)\right)}{2^{m \alpha}}+C \frac{\tilde{\rho}\left(2^{m+2}(|x-y| \vee|t-s|)\right)^{1+\alpha \gamma}}{2^{m \alpha} r^{\alpha(1+\gamma)}} \\
& \leq C \frac{\tilde{\rho}\left(2^{m+2}(|x-y| \vee|t-s|)\right)+\tilde{\rho}\left(2^{m+2}(|x-y| \vee|t-s|)\right)^{1+\alpha \gamma}}{2^{m \alpha}}
\end{aligned}
$$

Notice that

$$
\sup _{m \geq 1} \frac{\tilde{\rho}\left(2^{m+2} r\right)+\tilde{\rho}\left(2^{m+2} r\right)^{1+\alpha \gamma}}{2^{m \alpha}} \rightarrow 0 \quad \text { as } r \rightarrow 0 .
$$

Therefore, we can choose a modulus of continuity $\rho_{1}$ such that

$$
\rho_{1}(r) \geq C \sup _{m \geq 1} \frac{\tilde{\rho}\left(2^{m+2} r\right)+\tilde{\rho}\left(2^{m+2} r\right)^{1+\alpha \gamma}}{2^{m \alpha}} \text { for all } r \geq 0,
$$

and we have

$$
|u(y, s)-u(x, t)| \leq \rho_{1}(|x-y| \vee|t-s|) .
$$

If $|y-x| \geq r / 2$, then

$$
\begin{aligned}
|u(x, t)-u(y, s)| & \leq\left|u(x, t)-u\left(x_{0}, t\right)\right|+\left|u\left(x_{0}, t\right)-u(y, s)\right| \\
& \leq \tilde{\rho}(r)+\tilde{\rho}\left(\left|x_{0}-y\right| \vee|t-s|\right) \\
& \leq \tilde{\rho}(2(|x-y| \vee|t-s|))+\tilde{\rho}((|x-y|+r) \vee|t-s|) \\
& \leq \tilde{\rho}(2(|x-y| \vee|t-s|))+\tilde{\rho}(3(|x-y| \vee|t-s|)) \\
& \leq 2 \tilde{\rho}(3(|x-y| \vee|t-s|)) .
\end{aligned}
$$

If $|x-y| \leq r / 2$ and $|s-t| \geq r^{2+\gamma}(\tilde{\rho}(2 r))^{-\gamma} / 4$, then $r \leq 4^{\frac{1}{2+\gamma}}(2 M+2)^{\frac{\gamma}{2+\gamma}}|s-t|^{\frac{1}{2+\gamma}}$ when $\gamma \geq 0$, and $r \leq 2|s-t|^{\frac{1}{2}}$ when $\gamma \leq 0$. Then one can show similar to the above that

$$
\begin{aligned}
|u(x, t)-u(y, s)| & \leq 2 \tilde{\rho}\left(c\left(|x-y| \vee|t-s|^{\frac{1}{2}} \vee|s-t|^{\frac{1}{2+\gamma}}\right)\right) \\
& \leq \rho_{2}(|x-y| \vee|t-s|)
\end{aligned}
$$

where $\rho_{2}(r)=2 \tilde{\rho}\left(c r^{\frac{1}{2}}\right)$ or $\rho_{2}(r)=2 \tilde{\rho}\left(c r^{\frac{1}{2+\gamma}}\right)$ depending on whether $\gamma \geq 0$ or $\gamma \leq 0$ is a modulus of continuity, $c$ is a positive constant depending only on $M$ and $\gamma$. 
This finishes the proof in this first case.

Case 2: $r^{2+\gamma}(\tilde{\rho}(2 r))^{-\gamma} \geq 1+t$.

Then let $\lambda=\sqrt{|t+1|}$ when $\gamma \geq 0$, and $\lambda=(2 M+2)^{\frac{\gamma}{2+\gamma}}|t+1|^{\frac{1}{2+\gamma}}$ when $\gamma \in(-1,0)$. Then one can check that $\lambda \leq r$.

If $|y-x| \leq \lambda / 2$ and $|s-t| \leq \lambda^{2+\gamma}(\tilde{\rho}(2 \lambda))^{-\gamma} / 4$, let

$$
v(z, \tau)=\frac{u\left(\lambda z+x, \lambda^{2+\gamma}(\tilde{\rho}(2 \lambda))^{-\gamma} \tau+t\right)-u\left(x_{0}, t\right)}{\tilde{\rho}(2 \lambda)} \quad \text { for }(z, \tau) \in Q_{1} .
$$

Then

$$
v_{\tau}=\left(|\nabla v|^{2}+\varepsilon^{2} r^{2} \tilde{\rho}(2 \lambda)^{-2}\right)^{\gamma / 2}\left(\delta_{i j}+(p-2) \frac{v_{i} v_{j}}{|\nabla u|^{2}+\varepsilon^{2} \lambda^{2} \tilde{\rho}(2 \lambda)^{-2}}\right) u_{i j} \quad \operatorname{in} Q_{1} .
$$

Notice that $\lambda^{2+\gamma}(\tilde{\rho}(2 \lambda))^{-\gamma} \leq \lambda^{2} \leq \lambda$ when $\gamma \geq 0$, and $\lambda^{2+\gamma}(\tilde{\rho}(2 \lambda))^{-\gamma} \leq \lambda r^{1+\gamma}(\tilde{\rho}(2 r))^{-\gamma} \leq \lambda$ when $\gamma \in(-1,0)$. Thus, $|v(z, \tau)| \leq 1$ for $(z, \tau) \in Q_{1}$. Also, $\varepsilon \lambda / \tilde{\rho}(2 \lambda) \leq \varepsilon \lambda / \tilde{\rho}(\lambda) \leq \varepsilon<1$. Then, by the similar arguments in case 1 , we have

$$
|u(y, s)-u(x, t)| \leq \rho_{1}(|x-y| \vee|t-s|) .
$$

If $|y-x| \geq \lambda / 2$, then $|t+1| \leq c\left(|x-y|^{2} \vee|x-y|^{2+\gamma}\right) \leq c|x-y|$ for some $c>0$ depending only on $M$ and $\gamma$. Therefore,

$$
\begin{aligned}
|u(x, t)-u(y, s)| & \leq|u(x, t)-u(x,-1)|+|u(x,-1)-u(y, s)| \\
& \leq \tilde{\rho}(|t+1|)+\tilde{\rho}(|x-y| \vee|1+s|) \\
& \leq \tilde{\rho}(c|x-y|)+\tilde{\rho}((|x-y|) \vee|1+t|) \\
& \leq \tilde{\rho}(c(|x-y| \vee|t-s|))+\tilde{\rho}(c|x-y| \vee|t-s|) \\
& =2 \tilde{\rho}(c(|x-y| \vee|t-s|)) \\
& \leq \rho_{2}(|x-y| \vee|t-s|) .
\end{aligned}
$$

If $|x-y| \leq \lambda / 2$ and $|s-t| \geq \lambda^{2+\gamma}(\tilde{\rho}(2 \lambda))^{-\gamma} / 4$, then $\lambda \leq 4^{\frac{1}{2+\gamma}}(2 M+2)^{\frac{\gamma}{2+\gamma}}|s-t|^{\frac{1}{2+\gamma}}$ when $\gamma \geq 0$, and $\lambda \leq 2|s-t|^{\frac{1}{2}}$ when $\gamma \leq 0$. Then one can show similar to the above that

$$
\begin{aligned}
|u(x, t)-u(y, s)| & \leq|u(x, t)-u(x,-1)|+|u(x,-1)-u(y, s)| \\
& \leq \tilde{\rho}(|t+1|)+\tilde{\rho}(|x-y| \vee|1+s|) \\
& \leq \tilde{\rho}\left(c\left(|s-t|^{\frac{2}{2+\gamma}} \vee|s-t|^{\frac{2+\gamma}{2}}\right)\right)+\tilde{\rho}((|x-y|) \vee|1+t|) \\
& \leq \tilde{\rho}\left(c\left(|s-t|^{\frac{1}{2+\gamma}} \vee|s-t|^{\frac{1}{2}}\right)\right)+\tilde{\rho}\left(c\left(|s-t|^{\frac{1}{2+\gamma}} \vee|s-t|^{\frac{1}{2}}\right)\right) \\
& \leq \rho_{2}(|x-y| \vee|t-s|) .
\end{aligned}
$$

This finishes the proof in this second case.

In the end, we deal with the situation that $\gamma \in(-1,0)$ and $r^{1+\gamma}(2 M+2)^{-\gamma} \geq 1$. Then $r \geq c$ for $c=(2 M+2)^{\frac{\gamma}{1+\gamma}}$. Let $\lambda=(2 M+2)^{\frac{\gamma}{2+\gamma}}|t+1|^{\frac{1}{2+\gamma}}$. There exists $\mu>0$ depending only $M$ and $\gamma$ that if $|t+1| \leq \mu$, then $\lambda \leq c, c^{2+\gamma}(\tilde{\rho}(2 c))^{-\gamma} \geq 1+t$, and $\lambda^{1+\gamma}(2 M+2)^{-\gamma} \leq 1$. Then, for $t \leq-1+\mu$, the same arguments in case 2 works without any change. 
Now the final left case is that $(x, t) \in \bar{B}_{1-c} \times[-1+\mu, 0]$. Then we only need to consider that $(y, s) \in B_{1-c / 2} \times[-1+\mu / 2,0]$. It follows from Corollary 2.4 and Lemma 3.1 that there exists a modulus of continuity $\bar{\rho}$ depending only on $n, \gamma, p, M$ that

$$
u(x, t)-u(y, s) \mid \leq \bar{\rho}(|x-y| \vee|t-s|) .
$$

This finishes the final situation.

Then $\rho^{*}(r):=\rho_{1}(r)+\rho_{2}(r)+\bar{\rho}(r)$ is a desired modulus of continuity. The proof of this theorem is thereby completed.

\section{References}

[1] A. Banerjee and N. Garofalo. Gradient bounds and monotonicity of the energy for some nonlinear singular diffusion equations. Indiana Univ. Math. J., 62(2):699-736, 2013.

[2] A. Banerjee and N. Garofalo. Modica type gradient estimates for an inhomogeneous variant of the normalized $p$-Laplacian evolution. Nonlinear Anal., 121:458-468, 2015.

[3] A. Banerjee and N. Garofalo. On the Dirichlet boundary value problem for the normalized p-Laplacian evolution. Commun. Pure Appl. Anal., 14(1):1-21, 2015.

[4] G. Barles, S. Biton, and O. Ley. A geometrical approach to the study of unbounded solutions of quasilinear parabolic equations. Arch. Ration. Mech. Anal., 162(4):287-325, 2002.

[5] G. Barles and C. Georgelin. A simple proof of convergence for an approximation scheme for computing motions by mean curvature. SIAM J. Numer. Anal., 32(2):484-500, 1995.

[6] M. G. Crandall, M. Kocan, P. L. Lions, and A. Święch. Existence results for boundary problems for uniformly elliptic and parabolic fully nonlinear equations. Electron. J. Differential Equations, pages No. 24, 22 pp. 1999.

[7] E. DiBenedetto and A. Friedman. Hölder estimates for nonlinear degenerate parabolic systems. J. Reine Angew. Math., 357:1-22, 1985.

[8] K. Does. An evolution equation involving the normalized $p$-Laplacian. Commun. Pure Appl. Anal., 10(1):361-396, 2011.

[9] N. Garofalo. Unpublished notes. 1993.

[10] H. Ishii and P.-L. Lions. Viscosity solutions of fully nonlinear second-order elliptic partial differential equations. J. Differential Equations, 83(1):26-78, 1990.

[11] H. Ishii and P. Souganidis. Generalized motion of noncompact hypersurfaces with velocity having arbitrary growth on the curvature tensor. Tohoku Math. J. (2), 47(2):227-250, 1995.

[12] T. Jin and L. Silvestre. Hölder gradient estimates for parabolic homogeneous $p$-laplacian equations. to appear in J. Math. Pures Appl., May 2015. 
[13] P. Juutinen. Decay estimates in the supremum norm for the solutions to a nonlinear evolution equation. Proc. Roy. Soc. Edinburgh Sect. A, 144(3):557-566, 2014.

[14] B. Kawohl, S. Krömer, and J. Kurtz. Radial eigenfunctions for the game-theoretic $p$ Laplacian on a ball. Differential Integral Equations, 27(7-8):659-670, 2014.

[15] O. A. Ladyženskaja, V. A. Solonnikov, and N. N. Ural'ceva. Linear and quasilinear equations of parabolic type. Translated from the Russian by S. Smith. Translations of Mathematical Monographs, Vol. 23. American Mathematical Society, Providence, R.I., 1968.

[16] Q. Liu and A. Schikorra. General existence of solutions to dynamic programming equations. Commun. Pure Appl. Anal., 14(1):167-184, 2015.

[17] J. J. Manfredi, M. Parviainen, and J. D. Rossi. An asymptotic mean value characterization for a class of nonlinear parabolic equations related to tug-of-war games. SIAM J. Math. Anal., 42(5):2058-2081, 2010.

[18] J. J. Manfredi, M. Parviainen, and J. D. Rossi. Dynamic programming principle for tug-ofwar games with noise. ESAIM Control Optim. Calc. Var., 18(1):81-90, 2012.

[19] M. Ohnuma and K. Sato. Singular degenerate parabolic equations with applications to the p-Laplace diffusion equation. Comm. Partial Differential Equations, 22(3-4):381-411, 1997.

[20] Y. Peres and S. Sheffield. Tug-of-war with noise: a game-theoretic view of the $p$-Laplacian. Duke Math. J., 145(1):91-120, 2008.

[21] J. D. Rossi. Tug-of-war games and PDEs. Proc. Roy. Soc. Edinburgh Sect. A, 141(2):319$369,2011$.

[22] M. Rudd. Statistical exponential formulas for homogeneous diffusion. Commun. Pure Appl. Anal., 14(1):269-284, 2015.

[23] Y. Wang. Small perturbation solutions for parabolic equations. Indiana Univ. Math. J., 62(2):671-697, 2013.

[24] M. Wiegner. On $C_{\alpha}$-regularity of the gradient of solutions of degenerate parabolic systems. Ann. Mat. Pura Appl. (4), 145:385-405, 1986.

C. Imbert

Department of Mathematics and Applications, CNRS \& École Normale Supérieure (Paris)

45 rue d'Ulm, 75005 Paris, France

Email: Cyril.Imbert@ens.fr

T. Jin

Department of Mathematics, The Hong Kong University of Science and Technology

Clear Water Bay, Kowloon, Hong Kong

Email: tianlingjin@ust.hk 
L. Silvestre

Department of Mathematics, The University of Chicago

5734 S. University Avenue, Chicago, IL 60637, USA

Email: luis@math.uchicago.edu 